TRANSACTIONS OF THE

AMERICAN MATHEMATICAL SOCIETY

Volume 353, Number 8, Pages 3391-3408

S 0002-9947(01)02748-9

Article electronically published on April 9, 2001

\title{
VECTOR FIELDS WITH TOPOLOGICAL STABILITY
}

\author{
KAZUMINE MORIYASU, KAZUHIRO SAKAI, AND NAOYA SUMI
}

\begin{abstract}
In this paper, we give a characterization of the structurally stable vector fields by making use of the notion of topological stability. More precisely, it is proved that the $C^{1}$ interior of the set of all topologically stable $C^{1}$ vector fields coincides with the set of all vector fields satisfying Axiom A and the strong transversality condition.
\end{abstract}

\section{INTRODUCTION}

Let $M$ be a $C^{\infty}$ closed manifold and let $d$ be the distance on $M$ induced from a Riemannian metric $\|\cdot\|$ on the tangent bundle $T M$. Denote by $\mathcal{X}^{1}(M)$ the set of all $C^{1}$ vector fields on $M$ endowed with $C^{1}$ topology. Then every $X \in \mathcal{X}^{1}(M)$ generates a $C^{1}$ flow $X_{t}: M \times \mathbf{R} \rightarrow M$; that is a $C^{1}$ map such that $X_{t}: M \rightarrow M$ is a diffeomorphism satisfying $X_{0}(x)=x$ and $X_{t+s}(x)=X_{t}\left(X_{s}(x)\right)$ for all $s, t \in \mathbf{R}$ and $x \in M$. We say that $Y \in \mathcal{X}^{1}(M)$ is semiconjugate to $X \in \mathcal{X}^{1}(M)$ if $Y_{t}$ is semiconjugate to $X_{t}$; that is, there are a continuous surjection $h: M \rightarrow M$ and a continuous map $\tau: M \times \mathbf{R} \rightarrow \mathbf{R}$ such that

- for all $x \in M, \tau(x, 0)=0$ and $\tau(x, \cdot): \mathbf{R} \rightarrow \mathbf{R}$ is an orientation preserving homeomorphism,

- for all $x \in M$ and $t \in \mathbf{R}, h\left(Y_{t}(x)\right)=X_{\tau(x, t)}(h(x))$,

where $X_{t}$ and $Y_{t}$ are the flows induced by $X$ and $Y$ respectively. The pair $(h, \tau)$ is called a semiconjugacy from $Y$ to $X$. If $h$ can be taken as a homeomorphism, then we say that $Y$ is conjugate to $X$. A vector field $X \in \mathcal{X}^{1}(M)$ is called $\left(C^{1}\right)$ structurally stable if there is a $C^{1}$ neighborhood $\mathcal{U}(X)$ of $X$ in $\mathcal{X}^{1}(M)$ such that every $Y \in \mathcal{U}(X)$ is conjugate to $X$. It is proved by Robinson [1] that if $X$ satisfies Axiom $\mathrm{A}$ and the strong transversality condition, then $X$ is structurally stable.

Recently, Hayashi [4] proved a very important theorem on the $C^{1}$ connecting lemma (see also [17]) and by using this lemma, Hayashi [4] and Wen [16] gave a proof of $C^{1}$ stability conjecture for vector fields independently. After that Gan [3] gave another proof for the conjecture by combining with the connecting lemma and the methods in studying vector fields developed systematically by Liao 7 .

We shall also consider the $C^{0}$ topology on $\mathcal{X}^{1}(M)$. More precisely, for $X, Y \in$ $\mathcal{X}^{1}(M)$, let

$$
d_{C^{0}}(X, Y)=\sup _{x \in M}\{\|X(x)-Y(x)\|\} .
$$

Received by the editors October 12, 1999 and, in revised form, June 28, 2000.

2000 Mathematics Subject Classification. Primary 37C10, 37C15, 37C75, 37D20, 37D50; Secondary 37B99, 54H20.

Key words and phrases. Topologically stable, structurally stable, Axiom A, strong transversality condition, vector fields, flows. 
We say that $X \in \mathcal{X}^{1}(M)$ is topologically stable in $\mathcal{X}^{1}(M)$ if for any $\varepsilon>0$, there is $\delta>0$ such that for any $Y \in \mathcal{X}^{1}(M)$ with $d_{C^{0}}(X, Y)<\delta$, there is a semiconjugacy $(h, \tau)$ from $Y$ to $X$ satisfying $d(h(x), x)<\varepsilon$ for all $x \in M$ (see [6] and [15]).

The purpose of this paper is to give a characterization of the structurally stable vector fields by making use of the notion of topological stability. Denote by $\mathcal{T} \mathcal{S}(M)$ the set of all topologically stable $C^{1}$ vector fields on $M$. The following is proved.

Main Theorem. The $C^{1}$ interior of $\mathcal{T S}(M)$, int $\mathcal{T} \mathcal{S}(M)$, is characterized as the set of all vector fields satisfying Axiom $A$ and the strong transversality condition.

Our result includes the theorems proved by Hurley [6] and Wen [15. To prove the theorem we will check the Kupka-Smale condition (see [9]) for $X \in \operatorname{int} \mathcal{T} \mathcal{S}(M)$. For diffeomorphisms, the same result was proved by the first author 8] and the techniques used in there may be available to see the Kupka-Smale condition for periodic orbits (which are not singularities) of $X$. In this paper, we give a simpler proof of the transversality of the stable and the unstable manifolds of periodic orbits. In general, however, the techniques used in [8] cannot work for the proof of the hyperbolicity and the transversality of the stable and the unstable manifolds of singularities.

It follows immediately that if $X \in \mathcal{X}^{1}(M)$ satisfies Axiom A and the strong transversality condition, then $X$ is topologically stable in $\mathcal{X}^{1}(M)$ (see [12] and Remark stated below). As we have pointed out, the structural stable vector fields were characterized as the set of all vector fields satisfying Axiom A and the strong transversality condition. Thus, our theorem gives rise to the following

Corollary 1. For every $X \in \mathcal{X}^{1}(M), X$ is structurally stable if and only if $X \in$ $\operatorname{int} \mathcal{T} \mathcal{S}(M)$.

Recall that $X \in \mathcal{X}^{1}(M)$ is Morse-Smale (see [9]) if the non-wandering set is composed of a finite number of hyperbolic singularities and periodic orbits whose stable manifolds and unstable manifolds are all transversal. From [5, Theorem C], we see that if $X \in \mathcal{T S}(M)$ is $C^{0}$ approximated by a Morse-Smale vector field, then the non-wandering set of $X_{t}$ is composed of a finite number of singularities and periodic orbits. Since the set of all Morse-Smale vector fields is open and dense in $\mathcal{X}^{1}(M)$ when $M$ is a surface (see 9$]$ ), we have the following

Corollary 2. If $X \in \operatorname{int} \mathcal{T} \mathcal{S}(M)$ can be $C^{0}$ approximated by a Morse-Smale vector field, then $X$ is Morse-Smale. Especially, when $M$ is a surface, $\operatorname{int} \mathcal{T} \mathcal{S}(M)$ is characterized as the set of all Morse-Smale vector fields (cf. [1] and [14]).

Let $\mathcal{X}^{*}(M)$ be the set of all $X \in \mathcal{X}^{1}(M)$ with the property that there is a $C^{1}$ neighborhood $\mathcal{U}(X) \subset \mathcal{X}^{1}(M)$ of $X$ such that for every $Y \in \mathcal{U}(X)$, whose singularities and periodic orbits are hyperbolic. Denote by $\mathcal{X}^{\sharp}(M)$ all the systems $X \in \mathcal{X}^{*}(M)$ satisfying the following property: there is a $C^{1}$ neighborhood $\mathcal{U}(X) \subset$ $\mathcal{X}^{*}(M)$ of $X$ such that for each $Y \in \mathcal{U}(X)$, the stable manifolds and the unstable manifolds of singularities and periodic orbits of $Y_{t}$ are all transversal. Recently it is proved in [3] that $X \in \mathcal{X}^{\sharp}(M)$ if and only if $X$ satisfies Axiom A and the strong transversality condition. Thus $\mathcal{X}^{\sharp}(M) \subset \operatorname{int} \mathcal{T} \mathcal{S}(M)$. To get the main theorem it is enough to show that

Theorem. $\operatorname{int} \mathcal{T} \mathcal{S}(M)=\mathcal{X}^{\sharp}(M)$. 
Throughout this paper, let $\operatorname{Sing}(X)$ be the set of all singularities of $X \in \mathcal{X}^{1}(M)$, and let $P O\left(X_{t}\right)$ be the set of all periodic orbits (which are not singularities) of the integrated flow $X_{t}$. The proof of Theorem is divided into the following

Proposition A. $\operatorname{int} \mathcal{T} \mathcal{S}(M) \subset \mathcal{X}^{*}(M)$.

Proposition B. Let $X \in \operatorname{int} \mathcal{T} \mathcal{S}(M)$ and $p \in \operatorname{Sing}(X)$. Then, for every $\sigma \in$ Sing $(X) \cup P O\left(X_{t}\right)$, the stable manifold of $p$ and the unstable manifold of $\sigma$ are transverse.

Proposition C. Let $X \in \operatorname{int} \mathcal{T} \mathcal{S}(M)$ and let $\gamma, \gamma^{\prime} \in P O\left(X_{t}\right)$. Then the stable manifold of $\gamma$ and the unstable manifold of $\gamma^{\prime}$ are transverse.

When $M$ is a surface, if $\gamma \in P O\left(X_{t}\right)\left(X \in \mathcal{X}^{1}(M)\right)$ is hyperbolic, then $\gamma$ is an attractor or a repellor. Thus, in the last proposition, we may suppose that $\operatorname{dim} M \geq 3$.

Remark. Denote by $\mathcal{F}^{1}(M)$ the set of all $C^{1}$ flows on $M$ and topologize $\mathcal{F}^{1}(M)$ by using the $C^{0}$ topology on generating vector fields; that is, for $X_{t}, Y_{t} \in \mathcal{F}^{1}(M)$, let

$$
d_{C^{0}}^{\prime}\left(X_{t}, Y_{t}\right)=\sup _{x \in M}\left\{\left\|\dot{X}_{0}(x)-\dot{Y}_{0}(x)\right\|\right\} .
$$

Here $\dot{X}_{0}(x) \in T_{x} M$ is the tangent vector at $t=0$ to the curve $t \rightarrow X_{t}(x)$. We say that $X_{t} \in \mathcal{F}^{1}(M)$ is topologically stable in $\mathcal{F}^{1}(M)$ if for any $\varepsilon>0$, there is $\delta>0$ such that for any $Y_{t} \in \mathcal{F}^{1}(M)$ with $d_{C^{0}}^{\prime}\left(X_{t}, Y_{t}\right)<\delta$, there is a semiconjugacy $(h, \tau)$ from $Y_{t}$ to $X_{t}$ satisfying $d(h(x), x)<\varepsilon$ for all $x \in M$ (e.g. [1]). Robinson proved in 12] that if $X_{t} \in \mathcal{F}^{1}(M)$ satisfies Axiom A and the strong transversality condition (see [1] for the definitions), then $X_{t}$ is topologically stable in $\mathcal{F}^{1}(M)$.

Let $Y_{t} \in \mathcal{F}^{1}(M)$ be the flow induced by $Y \in \mathcal{X}^{1}(M)$ which satisfies Axiom A and the strong transversality condition. Then we see that $Y_{t}$ also satisfies Axiom A and the strong transversality condition so that $Y_{t}$ is topologically stable in $\mathcal{F}^{1}(M)$. Thus $Y$ is topologically stable in $\mathcal{X}^{1}(M)$.

\section{Preliminaries}

Let $M, d$ and $\mathcal{X}^{1}(M)$ be as before. In this paper, for $X, Y, \cdots \in \mathcal{X}^{1}(M)$, we denote the generated flows by $X_{t}, Y_{t}, \cdots$ respectively. Recall that $p \in \operatorname{Sing}(X)$ is hyperbolic if the linear map $D_{p} X: T_{p} M \rightarrow T_{p} M$ has no eigenvalue $\lambda$ with $\operatorname{Re}(\lambda)=0$ (e.g. 9 p.58]). For a hyperbolic singularity $p$, we define the stable manifold $W^{s}\left(p, X_{t}\right)$ and the unstable manifold $W^{u}\left(p, X_{t}\right)$ of $p$ as usual. A point $x \in M$ is called a non-wandering point of $X$ if for any neighborhood $U$ of $x$ in $M$, there is $t \geq 1$ such that $X_{t}(U) \cap U \neq \emptyset$. The set of all non-wandering points of $X$ is denoted by $\Omega\left(X_{t}\right)$. Clearly, $\operatorname{Sing}(X) \cup P O\left(X_{t}\right) \subset \Omega\left(X_{t}\right)$. We say that $X$ satisfies Axiom $A$ if $P O\left(X_{t}\right)$ is dense in $\Omega\left(X_{t}\right) \backslash \operatorname{Sing}(X)$, and if there are constants $C>0$ and $\lambda>0$ such that the tangent flow $D X_{t}: T M \rightarrow T M$ leaves invariant a continuous splitting $T_{\Omega\left(X_{t}\right)} M=E^{s} \oplus \operatorname{Span} X \oplus E^{u}$ such that

$$
\left\|D X_{t_{\mid E^{s}(x)}}\right\| \leq C e^{-\lambda t} \text { and }\left\|D X_{-t_{\mid E^{u}(x)}}\right\| \leq C e^{-\lambda t} .
$$

for $t>0$ and $x \in \Omega\left(X_{t}\right)$.

Hereafter, we assume that the exponential map $\exp _{p}: T_{p} M(1) \rightarrow M$ is well defined for all $p \in M$, where $T_{p} M(1)=\left\{v \in T_{p} M:\|v\| \leq 1\right\}$. Let $B_{\varepsilon}(x)=\{y \in$ $M: d(x, y) \leq \varepsilon\}(\varepsilon>0)$. Then, by mimicking the proof of [2, Lemma 1.1], we 
have the following being used in the proof of the hyperbolicity of the singularity in section 2 .

Lemma 1.1. Let $X \in \mathcal{X}^{1}(M)$ and $p \in \operatorname{Sing}(X)$. Then for every $C^{1}$ neighborhood $\mathcal{U}(X) \subset \mathcal{X}^{1}(M)$ of $X$, there are $\delta_{0}>0$ and $\varepsilon_{0}>0$ such that if $\mathcal{O}_{\delta}: T_{p} M \rightarrow T_{p} M$ is a linear map with $\left\|\mathcal{O}_{\delta}-D_{p} X\right\|<\delta<\delta_{0}$, then there is $Y^{\delta} \in \mathcal{U}(X)$ satisfying

$$
Y^{\delta}(x)= \begin{cases}\left(D_{\exp _{p}^{-1}(x)} \exp _{p}\right) \circ \mathcal{O}_{\delta} \circ \exp _{p}^{-1}(x) & \text { if } x \in B_{\varepsilon_{0} / 4}(p), \\ X(x) & \text { if } x \notin B_{\varepsilon_{0}}(p) .\end{cases}
$$

Furthermore, $d_{C^{0}}\left(Y^{\delta}, Y^{0}\right) \rightarrow 0$ as $\delta \rightarrow 0$. Here $Y^{0}$ is the vector field for $\mathcal{O}_{0}=D_{p} X$.

Remark 1.1. By Lemma 1.1, $Y_{\mid B_{\varepsilon_{0} / 4}(p)}$ is regarded as a linearization of $X_{\mid B_{\varepsilon_{0} / 4}}(p)$ with respect to the exponential coordinates. If there are an interval $I \subset \mathbf{R}$ and an integral curve $\xi(t)(t \in I)$ of the linear vector field $\mathcal{O}_{\delta}$ in $\exp _{p}^{-1}\left(B_{\varepsilon_{0} / 4}(p)\right) \subset T_{p} M$, then the composition $\exp _{p} \circ \xi: I \rightarrow M$ is an integral curve of $Y^{\delta}$ in $B_{\varepsilon_{0} / 4}(p) \subset M$ (e.g. [9] p.10]).

Let $X \in \mathcal{X}^{1}(M)$. For every $x \in M \backslash \operatorname{Sing}(X)$, put $\hat{\Pi}_{x}=(\operatorname{Span} X(x))^{\perp} \subset T_{x} M$ (see [10, p.266]), $\Pi_{x, r}=\exp _{x}\left(\hat{\Pi}_{x, r}\right)$ and $\Pi_{x}=\Pi_{x, 1}$, where $\hat{\Pi}_{x, r}=\left\{v \in \hat{\Pi}_{x}:\|v\|<\right.$ $r\}$ for $r>0$. Then, for given $x^{\prime}=X_{t_{0}}(x)\left(t_{0}>0\right)$, there are $r_{0}>0$ and a $C^{1}$ map $\tau: \Pi_{x, r_{0}} \rightarrow \mathbf{R}$ such that $X_{\tau(y)}(y) \in \Pi_{x^{\prime}}\left(y \in \Pi_{x, r_{0}}\right)$ with $\tau(x)=t_{0}$. The flow $X_{t}$ uniquely defines the Poincaré map $f: \Pi_{x, r_{0}} \rightarrow \Pi_{x^{\prime}}$ by $f(y)=X_{\tau(y)}(y)$ for all $y \in \Pi_{x, r_{0}}$. The map is $C^{1}$ embedding whose image is interior to $\Pi_{x^{\prime}}$ if $r_{0}$ is small. We denote the set of all $C^{1}$ embeddings from $\Pi_{x, r}$ to $\Pi_{x^{\prime}}(r>0)$ by $E m b^{1}\left(\Pi_{x, r}, \Pi_{x^{\prime}}\right)$ and topologize it by using the $C^{1}$ topology. If $X_{t}(x) \neq x$ for $0<t \leq t_{0}$ and $r_{0}$ is sufficiently small, then $(t, y) \mapsto X_{t}(y) C^{1}$ embeds

$$
\left\{(t, y) \in \mathbf{R} \times \Pi_{x, r}: 0 \leq t \leq \tau(y)\right\}
$$

for $0<r \leq r_{0}$. The image

$$
\left\{X_{t}(y): y \in \Pi_{x, r} \text { and } 0 \leq t \leq \tau(y)\right\}
$$

is called a $t_{0}$-time length flow box and is denoted by $F_{x}\left(X_{t}, r, t_{0}\right)$. For $\varepsilon>0$, let $\mathcal{N}_{\varepsilon}\left(\Pi_{x, r}\right)$ be the set of all diffeomorphisms $\varphi: \Pi_{x, r} \rightarrow \Pi_{x, r}$ such that $\operatorname{supp}(\varphi) \subset$ $\Pi_{x, r / 2}$ and $d_{C^{1}}(\varphi, i d)<\varepsilon$. Here $d_{C^{1}}$ is the usual $C^{1}$ metric, $i d: \Pi_{x, r} \rightarrow \Pi_{x, r}$ is the identity map and the support of $\varphi$ is the closure of the set where it differs from $i d$.

Lemma 1.2. Let $X \in \mathcal{X}^{1}(M)$. Suppose $X_{t}(x) \neq x$ for $0<t \leq t_{0}(x \notin \operatorname{Sing}(X))$, and let $f: \Pi_{x, r_{0}} \rightarrow \Pi_{x^{\prime}}\left(x^{\prime}=X_{t_{0}}(x)\right)$ be the Poincaré map $\left(r_{0}>0\right.$ is sufficiently small). Then, for every $C^{1}$ neighborhood $\mathcal{U}(X) \subset \mathcal{X}^{1}(M)$ of $X$ and $0<r \leq r_{0}$, there is $\varepsilon>0$ with the property that for every $\varphi \in \mathcal{N}_{\varepsilon}\left(\Pi_{x, r}\right)$, there exists $Y \in \mathcal{U}(X)$ satisfying

$$
\begin{cases}Y(y)=X(y) & \text { if } x \notin F_{x}\left(X_{t}, r, t_{0}\right), \\ f_{Y}(y)=f \circ \varphi(y) & \text { if } y \in \Pi_{x, r} .\end{cases}
$$

Here $f_{Y}: \Pi_{x, r} \rightarrow \Pi_{x^{\prime}}$ is the Poincaré map defined by $Y_{t}$.

Proof. See [10, p.296, Remark 2].

Remark 1.2. Under the same notation and assumption of Lemma 1.2, let $Y^{\delta} \in$ $\mathcal{U}(X)$ be given by Lemma 1.2 for $\varphi_{\delta} \in \mathcal{N}_{\varepsilon}\left(\Pi_{x, r}\right)(\delta>0)$. If $\varphi_{\delta} \rightarrow \varphi$ as $\delta \rightarrow 0$ with respect to the $C^{1}$ topology, then, by the construction of $Y^{\delta}$ stated in the proof [10. p.296], it can be checked that $d_{C^{0}}\left(Y^{\delta}, Y\right) \rightarrow 0$ as $\delta \rightarrow 0$. 
Let $X \in \mathcal{X}^{1}(M)$ and suppose $p \in \gamma \in P O\left(X_{t}\right)\left(X_{T}(p)=p, T>0\right)$. If $f$ : $\Pi_{p, r_{0}} \rightarrow \Pi_{p}$ is the Poincaré map $\left(r_{0}>0\right)$, then $f(p)=p$. We say that $\gamma$ is hyperbolic if $p$ is a hyperbolic fixed point of $f$ (e.g. [9, p.95]). If $\gamma \in P O\left(X_{t}\right)$ is hyperbolic, then the stable manifold $W^{s}\left(\gamma, X_{t}\right)$ and the unstable manifold $W^{u}\left(\gamma, X_{t}\right)$ of $\gamma$ are defined by the usual way. Let $\gamma, \gamma^{\prime} \in P O\left(X_{t}\right)$ be hyperbolic. We say that $\gamma$ is transverse to $\gamma^{\prime}$ if for any $x \in W^{s}\left(\gamma, X_{t}\right) \cap W^{u}\left(\gamma^{\prime}, X_{t}\right)$,

$$
T_{x} M=T_{x} W^{s}\left(\gamma, X_{t}\right)+T_{x} W^{u}\left(\gamma^{\prime}, X_{t}\right) .
$$

The following lemma plays an essential role in the proof of the hyperbolicity of the periodic orbits and in the proof of the transversality of the stable and the unstable manifolds (see sections 2 and 3 ).

Lemma 1.3. Let $X \in \mathcal{X}^{1}(M), p \in \gamma \in P O\left(X_{t}\right)\left(X_{T}(p)=p\right)$ and $f: \Pi_{p, r_{0}} \rightarrow \Pi_{p}$ be as above, and let $\mathcal{U}(X) \subset \mathcal{X}^{1}(M)$ be a $C^{1}$ neighborhood of $X$ and $0<r \leq r_{0}$ be given. Then there are $\delta_{0}>0$ and $0<\varepsilon_{0}<r / 2$ such that for a linear isomorphism $\mathcal{O}_{\delta}: \hat{\Pi}_{p} \rightarrow \hat{\Pi}_{p}$ with $\left\|\mathcal{O}_{\delta}-D_{p} f\right\|<\delta<\delta_{0}$, there is $Y^{\delta} \in \mathcal{U}(X)$ satisfying

(i) $Y^{\delta}(x)=X(x)$ if $x \notin F_{p}\left(X_{t}, r, T\right)$,

(ii) $p \in \gamma \in P O\left(Y_{t}^{\delta}\right)$,

(iii) $g_{Y^{\delta}}(x)= \begin{cases}\exp _{p} \circ \mathcal{O}_{\delta} \circ \exp _{p}^{-1}(x) & \text { if } x \in B_{\varepsilon_{0} / 4}(p) \cap \Pi_{p, r}, \\ f(x) & \text { if } x \notin B_{\varepsilon_{0}}(p) \cap \Pi_{p, r}\end{cases}$

where $g_{Y^{\delta}}: \Pi_{p, r} \rightarrow \Pi_{p}$ is the Poincaré map of $Y_{t}^{\delta}$. Furthermore, let $Y^{0}$ be the vector field for $\mathcal{O}_{0}=D_{p} f$. Then we have

(iv) $d_{C^{0}}\left(Y^{\delta}, Y^{0}\right) \rightarrow 0$ as $\delta \rightarrow 0$.

Proof. Let $\tau: \Pi_{p, r_{0}} \rightarrow \mathbf{R}$ be the $C^{1}$ function such that $f(x)=X_{\tau(x)}(x) \in \Pi_{p}$ $\left(x \in \Pi_{p, r_{0}}\right)$. Put $p^{\prime}=X_{T / 2}(p)$, and for $x \in \Pi_{p, r_{0}}$, take $0<\tau^{\prime}(x)<T$ such that $X_{\tau^{\prime}(x)}(x) \in \Pi_{p^{\prime}}$. Clearly $\tau^{\prime}: \Pi_{p, r_{0}} \rightarrow \mathbf{R}$ is a $C^{1}$ function. Set $f^{\prime}(x)=X_{\tau^{\prime}(x)}(x) \in$ $\Pi_{p^{\prime}}\left(x \in \Pi_{p, r_{0}}\right)$. Then $f^{\prime}: \Pi_{p, r_{0}} \rightarrow \Pi_{p^{\prime}}$ is a $C^{1}$ embedding. We apply Lemma 1.2 to $X$ and $f^{\prime}$. For every $C^{1}$ neighborhood $\mathcal{U}(X)$ of $X$ and $0<r \leq r_{0}$, let $\varepsilon>0$ be given by Lemma 1.2. Choose a $C^{1}$ neighborhood $\mathcal{U}(f) \subset E m b^{1}\left(\Pi_{p, r}, \Pi_{p}\right)$ of $f$ such that if

- $g \in \mathcal{U}(f)$,

- $g(p)=p$ and $g(x)=f(x)$ when $x \notin \Pi_{p, r / 2}$,

then, $\varphi_{g}=f^{-1} \circ g \in \mathcal{N}_{\varepsilon}\left(\Pi_{p, r}\right)$. It is easy to see that $X_{\tau\left(\varphi_{g}(x)\right)}\left(\varphi_{g}(x)\right)=g(x)$ for $x \in \Pi_{p, r}$ (recall $f(p)=p$ ). By [2, Lemma 1.1], there are $\delta_{0}>0$ and $0<\varepsilon_{0}<r / 2$ such that if $\mathcal{O}_{\delta}: \hat{\Pi}_{p} \rightarrow \hat{\Pi}_{p}$ is a linear map with $\left\|\mathcal{O}_{\delta}-D_{p} f\right\|<\delta<\delta_{0}$, then there is $g_{\delta} \in \mathcal{U}(f)$ satisfying

$$
g_{\delta}(x)= \begin{cases}\exp _{p} \circ \mathcal{O}_{\delta} \circ \exp _{p}^{-1}(x) & \text { if } x \in B_{\varepsilon_{0} / 4}(p) \cap \Pi_{p, r}, \\ f(x) & \text { if } x \notin B_{\varepsilon_{0}}(p) \cap \Pi_{p, r}\end{cases}
$$

and $d_{C^{1}}\left(g_{\delta}, g_{0}\right) \rightarrow 0$ as $\delta \rightarrow 0$. Here $g_{0}$ is the map for $\mathcal{O}_{0}\left(=D_{p} f\right)$. If we put $\varphi_{g_{\delta}}=$ $f^{-1} \circ g_{\delta}$, then, by the choice of $\mathcal{U}(f)$, there are $Y^{\delta} \in \mathcal{U}(X)$ and $f_{Y^{\delta}}: \Pi_{p, r} \rightarrow \Pi_{p^{\prime}}$ (induced by $Y_{t}^{\delta}$ ) such that

$$
\begin{cases}Y^{\delta}(x)=X(x) & \text { if } x \notin F_{p}\left(X_{t}, r, T / 2\right), \\ f_{Y^{\delta}}(x)=f^{\prime} \circ \varphi_{g_{\delta}}(x)=X_{\tau^{\prime}\left(\varphi_{g_{\delta}}(x)\right)}\left(\varphi_{g_{\delta}}(x)\right) & \text { if } x \in \Pi_{p, r} .\end{cases}
$$

Denote $X_{\tau\left(\varphi_{g_{\delta}}(x)\right)-\tau^{\prime}\left(\varphi_{g_{\delta}}(x)\right)}\left(f_{Y^{\delta}}(x)\right)$ by $g_{Y^{\delta}}(x)$ for $x \in \Pi_{p, r}$. Then

$$
g_{Y^{\delta}}(x)=X_{\tau\left(\varphi_{g_{\delta}}(x)\right)}\left(\varphi_{g_{\delta}}(x)\right)=g_{\delta}(x)
$$


for $x \in \Pi_{p, r}$. Let $Y^{0}$ be the vector field for $\mathcal{O}_{0}$. Then, by Remark 1.2, $d_{C^{0}}\left(Y^{\delta}, Y^{0}\right)$ $\rightarrow 0$ as $\delta \rightarrow 0$ (since $d_{C^{1}}\left(g_{\delta}, g_{0}\right) \rightarrow 0$ as $\left.\delta \rightarrow 0\right)$.

\section{Proof of Proposition A}

At first, we show the conclusion for singularities. Let $\mathcal{T S}(M)$ be as before and fix $X \in \operatorname{int} \mathcal{T} \mathcal{S}(M)$. Suppose that there is an eigenvalue $\lambda$ of $D_{p} X$ with $\operatorname{Re}(\lambda)=0$ for some $p \in \operatorname{Sing}(X)$. By Lemma 1.1, for any $C^{1}$ neighborhood $\mathcal{U}(X) \subset \mathcal{T S}(M)$ of $X$, there are $\delta_{0}>0$ and $\varepsilon_{0}>0$ such that for every linear isomorphism $\mathcal{O}_{\delta}: T_{p} M \rightarrow T_{p} M$ with $\left\|\mathcal{O}_{\delta}-D_{p} X\right\|<\delta<\delta_{0}$, there is $Y^{\delta} \in \mathcal{U}(X)$ satisfying

$$
Y^{\delta}(x)= \begin{cases}\left(D_{\exp _{p}^{-1}(x)} \exp _{p}\right) \circ \mathcal{O}_{\delta} \circ \exp _{p}^{-1}(x) & \text { if } x \in B_{\varepsilon_{0} / 4}(p) \\ X(x) & \text { if } x \notin B_{\varepsilon_{0}}(p) .\end{cases}
$$

Let $Y^{0} \in \mathcal{U}(X)$ be as above for $\mathcal{O}_{0}=D_{p} X$ and denote $Y^{0}$ by $Y$. For $0<\varepsilon<\varepsilon_{0} / 16$, let $0<\delta<\min \left\{\delta_{0}, \varepsilon\right\}$ be as in the definition of the topological stability of $Y_{t}$. Pick $0<\delta^{\prime}<\delta$ and a linear isomorphism $\mathcal{O}_{\delta^{\prime}}: T_{p} M \rightarrow T_{p} M$ whose any eigenvalue has a non-zero real part such that $d_{C^{0}}\left(Y, Y^{\delta^{\prime}}\right)<\delta$. Then $p$ is a hyperbolic singularity of $Y^{\delta^{\prime}}$. By Remark 1.1, the restriction $Y_{t}^{\delta^{\prime}}{ }_{\mid B_{\varepsilon_{0} / 4}(p)}$ can be regarded as the flow induced from the hyperbolic linear vector field $\mathcal{O}_{\delta^{\prime}} \mid \exp _{p}^{-1}\left(B_{\varepsilon_{0} / 4}(p)\right)$ with respect to the exponential coordinates. Since $d_{C^{0}}\left(Y, Y^{\delta^{\prime}}\right)<\delta$, there are $h: M \rightarrow M$ and $\tau: M \times \mathbf{R} \rightarrow \mathbf{R}$ such that $h\left(Y_{t}^{\delta^{\prime}}(x)\right)=Y_{\tau(x, t)}(h(x))$ for all $x \in M$ and $d(h, i d)<\varepsilon$. By the existence of the $\lambda$, we can take $z \in M$ such that

$$
p \notin B_{\varepsilon}\left(Y_{\mathbf{R}}(z)\right) \subset B_{\varepsilon_{0} / 8}(p)
$$

(by reducing $\varepsilon$ if necessary). Here $Y_{\mathbf{R}}(z)=\left\{Y_{t}(z): t \in \mathbf{R}\right\}$ and $B_{\varepsilon}(A)=$ $\bigcup_{x \in A} B_{\varepsilon}(x)$ for $A \subset M$. Fix $w \in h^{-1}(z)$. Then, since $h\left(Y_{t}^{\delta^{\prime}}(w)\right)=Y_{\tau(w, t)}(z)$ and $d(h, i d)<\varepsilon$, we have $Y_{\mathbf{R}}^{\delta^{\prime}}(w) \subset B_{\varepsilon}\left(Y_{\mathbf{R}}(z)\right)$. This is a contradiction since $Y_{t}^{\delta^{\prime}}{ }_{\mid B_{\varepsilon_{0} / 4}(p)}$ is regarded as the flow induced from the hyperbolic linear vector field $\mathcal{O}_{\delta^{\prime} \mid \exp _{p}^{-1}\left(B_{\varepsilon_{0} / 4}(p)\right)}$.

Next we prove the proposition for periodic orbits. Let $\mathcal{U}(X) \subset \mathcal{T} \mathcal{S}(M)$ be a $C^{1}$ neighborhood of $X$ and pick $p \in \gamma \in P O\left(X_{t}\right)\left(X_{T}(p)=p, T>0\right)$. The flow $X_{t}$ defines the Poincaré map $f: \Pi_{p, r_{0}} \rightarrow \Pi_{p}$ (for some $r_{0}>0$ ). By assuming that there is an eigenvalue $\lambda$ of $D_{p} f$ with $|\lambda|=1$, we shall derive a contradiction. Let $\delta_{0}>0$ and $0<\varepsilon_{0}<r_{0}$ be given by Lemma 1.3 for the $\mathcal{U}(X)$. Then, for every linear isomorphism $\mathcal{O}_{\delta}: \hat{\Pi}_{p} \rightarrow \hat{\Pi}_{p}$ with $\left\|\mathcal{O}_{\delta}-D_{p} f\right\|<\delta<\delta_{0}$, there is $Y^{\delta} \in \mathcal{U}(X)$ such that

- $Y^{\delta}(x)=X(x)$ if $x \notin F_{p}\left(X_{t}, r_{0}, T\right)$,

- $g_{Y^{\delta}}(x)= \begin{cases}\exp _{p} \circ \mathcal{O}_{\delta} \circ \exp _{p}^{-1}(x) & \text { if } x \in B_{\varepsilon_{0} / 4}(p) \cap \Pi_{p, r_{0}}, \\ f(x) & \text { if } x \notin B_{\varepsilon_{0}}(p) \cap \Pi_{p, r_{0}},\end{cases}$

- $d_{C^{0}}\left(Y^{\delta}, Y^{0}\right) \rightarrow 0$ as $\delta \rightarrow 0$.

Denote $Y^{0}$ by $Y$. For $0<\varepsilon<\varepsilon_{0} / 16$, let $0<\delta<\min \left\{\delta_{0}, \varepsilon\right\}$ be as in the definition of the topological stability of $Y_{t}$. Take $0<\delta^{\prime}<\delta$ and a hyperbolic linear isomorphism $\mathcal{O}_{\delta^{\prime}}: \hat{\Pi}_{p} \rightarrow \hat{\Pi}_{p}$ with $d_{C^{0}}\left(Y, Y^{\delta^{\prime}}\right)<\delta$. Then there are $h: M \rightarrow M$ and $\tau:$ $M \times \mathbf{R} \rightarrow \mathbf{R}$ satisfying $h\left(Y_{t}^{\delta^{\prime}}(x)\right)=Y_{\tau(x, t)}(h(x))$ for all $x \in M$ and $d(h, i d)<$ $\varepsilon$. Remark that $g_{Y^{\delta^{\prime}}}(p)=p$ and the restriction $g_{Y^{\delta^{\prime}} \mid B_{\varepsilon_{0} / 4}(p) \cap \Pi_{p, r_{0}}}$ is regarded 
as the hyperbolic linear isomorphism $\mathcal{O}_{\delta^{\prime}} \mid \exp _{p}^{-1}\left(B_{\varepsilon_{0} / 4}(p) \cap \Pi_{p, r_{0}}\right)$ with respect to the exponential coordinates. Since $|\lambda|=1$, we may take $z \in \Pi_{p, r_{0}}$ such that

$$
p \notin B_{\varepsilon}\left(\left\{g_{Y}^{i}(z): i \in \mathbf{Z}\right\}\right) \subset B_{\varepsilon_{0} / 8}(p) \cap \Pi_{p, r_{0}} .
$$

Fix $w \in h^{-1}(z)$ and set $w^{\prime}=Y_{t^{\prime}}^{\delta^{\prime}}(w) \in \Pi_{p, r_{0}}$, where $\left|t^{\prime}\right|=\min \left\{|t|: Y_{t}^{\delta^{\prime}}(w) \in\right.$ $\left.\Pi_{p, r_{0}}\right\}$. Then, since $h\left(Y_{t}^{\delta^{\prime}}(w)\right)=Y_{\tau(w, t)}(z)$ and $d(h, i d)<\varepsilon$, we have $g_{Y^{\delta^{\prime}}}^{i}\left(w^{\prime}\right) \in$ $B_{\varepsilon}\left(\left\{g_{Y}^{i}(z): i \in \mathbf{Z}\right\}\right)$ for all $i \in \mathbf{Z}$. This is a contradiction since $g_{Y^{\delta^{\prime}} \mid B_{\varepsilon_{0} / 4}(p) \cap \Pi_{p, r_{0}}}$ can be regarded as the hyperbolic linear isomorphism. The proposition is proved.

\section{Proof of Proposition B}

Let $\mathcal{T} \mathcal{S}(M)$ be as before and fix $X \in \operatorname{int} \mathcal{T} \mathcal{S}(M)$. Suppose that $x \in W^{s}\left(p, X_{t}\right) \cap$ $W^{u}\left(\sigma, X_{t}\right)\left(p \in \operatorname{Sing}(X), \sigma \in \operatorname{Sing}(X) \cup P O\left(X_{t}\right)\right)$ and

$$
T_{x} M \neq T_{x} W^{s}\left(p, X_{t}\right)+T_{x} W^{u}\left(\sigma, X_{t}\right) .
$$

We may assume that $x$ is very near $p$. Take $r_{0}>0$ small enough so that

- there are the Poincaré maps $f: \Pi_{x, r_{0}} \rightarrow \Pi_{X_{1}(x)}$ and $f^{\prime}: \Pi_{X_{-1}(x), r_{0}} \rightarrow \Pi_{x}$,

- $\left\{X_{t}(x): t<0\right\} \cap \Pi_{x, r_{0}}=\emptyset$ and $\left\{X_{t}(x): t<-1\right\} \cap \Pi_{X_{-1}(x), r_{0}}=\emptyset$,

- $W_{2 r_{0}}^{u}\left(\sigma, X_{t}\right) \cap\left(\Pi_{x, r_{0}} \cup \Pi_{X_{-1}(x), r_{0}}\right)=\emptyset$.

Here $W_{2 r_{0}}^{u}\left(\sigma, X_{t}\right)$ is the local unstable manifold of $\sigma$. Put

$V^{s}(x)=$ the connected component of $W^{s}\left(p, X_{t}\right) \cap \Pi_{x}$ containing $x$,

$V^{u}(x)=$ the connected component of $W^{u}\left(\sigma, X_{t}\right) \cap \Pi_{x}$ containing $x$.

Clearly, $0 \leq \operatorname{dim} V^{s}(x) \leq \operatorname{dim} \Pi_{x}$ and $0 \leq \operatorname{dim} V^{u}(x) \leq \operatorname{dim} \Pi_{x}$. If

$$
\operatorname{dim} V^{s}(x)=\operatorname{dim} \Pi_{x} \text { or } \operatorname{dim} V^{u}(x)=\operatorname{dim} \Pi_{x},
$$

then there is nothing to prove. Notice that $\hat{\Pi}_{x} \neq T_{x} V^{s}(x)+T_{x} V^{u}(x)$. Finally, fix $t_{1}>0$ such that $X_{-t_{1}}(x) \in W_{r_{0}}^{u}\left(\sigma, X_{t}\right)$.

We shall divide the proof into the following two cases:

Case 1. $\operatorname{dim} V^{s}(x)+\operatorname{dim} V^{u}(x)<\operatorname{dim} \Pi_{x}$,

Case 2. $\operatorname{dim} V^{s}(x)+\operatorname{dim} V^{u}(x) \geq \operatorname{dim} \Pi_{x}$.

An outline of the proof is, roughly speaking, with a small perturbation we destroy the intersection of two manifolds $V^{s}(x)$ and $V^{u}(x)$ and derive a contradiction in Case 1 (see Figure 1). In Case 2, at first, we attach $V^{s}(x)$ and $V^{u}(x)$ in a small neighborhood of $x$ (see Figure 2) and next, we destroy the intersection "locally" with small perturbations (see Figure 3 ). Then we shall derive a contradiction.

First of all, we make use of Lemma 1.2 for $X$ and $f$. For $\delta>0$, let $\varepsilon(\delta)>0$ be the number such that if $\varphi \in \mathcal{N}_{\varepsilon(\delta)}\left(\Pi_{x, r_{0}}\right)$, then the corresponding vector field $Y$ given by Lemma 1.2 satisfies $d_{C^{0}}(X, Y)<\delta$. Put

$$
V_{r}^{u}(x)=\text { the connected component of } V^{u}(x) \cap B_{r}(x) \text { containing } x
$$

for $r>0$. If $\operatorname{dim} V^{s}(x)+\operatorname{dim} V^{u}(x)<\operatorname{dim} \Pi_{x}$ (Case 1 ), then the following is easily obtained: there is $0<r^{\prime}<r_{0} / 4$ with the property that

(1.1) for every $\delta>0$, there exists $\varphi_{\delta} \in \mathcal{N}_{\varepsilon(\delta)}\left(\Pi_{x, r_{0}}\right)$ satisfying $\varphi_{\delta}\left(V_{r^{\prime}}^{u}(x)\right) \cap$ $V^{s}(x)=\emptyset$,

(1.2) $V_{r^{\prime}}^{u}(x)=\left[\bigcup_{0 \leq t \leq t_{1}} X_{t}\left(W_{2 r_{0}}^{u}\left(\sigma, X_{t}\right)\right)\right] \cap \Pi_{x, r_{0}} \cap B_{r^{\prime}}(x)$,

(1.3) $B_{r^{\prime}}\left(\left\{X_{t}(x): t \leq 0\right\}\right) \cap \Pi_{x, r_{0}}=B_{r^{\prime}}(x) \cap \Pi_{x, r_{0}} \quad$ (see Figure 1). 


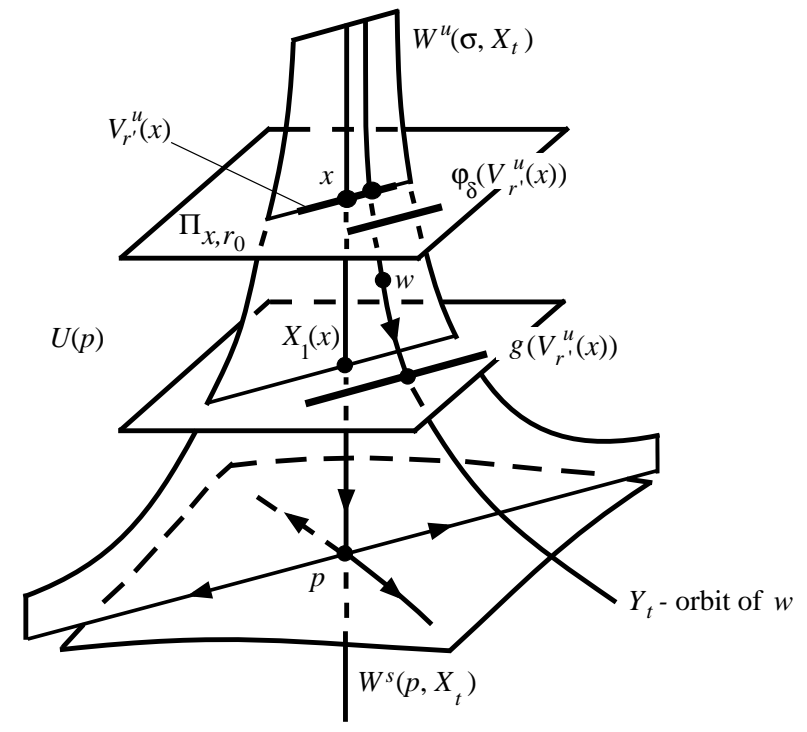

FIGURE 1.

For a sufficiently small $0<\varepsilon<r^{\prime}$, pick $0<\delta<\varepsilon$ as in the definition of the topological stability of $X_{t}$. Let $Y^{\delta}$ be the vector field given by Lemma 1.2 for the above perturbation (1.1); that is,

$$
\begin{cases}Y^{\delta}(y)=X(y) & \text { if } y \notin F_{x}\left(X_{t}, r_{0}, 1\right), \\ g(y)=f \circ \varphi_{\delta}(y) & \text { if } y \in \Pi_{x, r_{0}} \\ d_{C^{0}}\left(X, Y^{\delta}\right)<\delta, & \end{cases}
$$

where $g: \Pi_{x, r_{0}} \rightarrow \Pi_{X_{1}(x)}$ is the Poincaré map induced by $Y_{t}^{\delta}$. For simplicity, denote $Y^{\delta}$ by $Y$. Then, since $d_{C^{0}}(X, Y)<\delta$, there are $h: M \rightarrow M$ and $\tau: M \times \mathbf{R} \rightarrow \mathbf{R}$ such that $h\left(Y_{t}(y)\right)=X_{\tau(y, t)}(h(y))$ for all $y \in M$ and $d(h, i d)<\varepsilon$. Remark that

$$
\left(\left\{X_{t}(x): t<0\right\} \cup W_{2 r_{0}}^{u}\left(\sigma, X_{t}\right)\right) \cap \Pi_{x, r_{0}}=\emptyset
$$

and $Y(y)=X(y)$ if $y \notin F_{x}\left(X_{t}, r_{0}, 1\right)$. Thus $\sigma$ is also a hyperbolic singularity or periodic orbit of $Y$. Especially, $W_{2 r_{0}}^{u}\left(\sigma, X_{t}\right)=W_{2 r_{0}}^{u}\left(\sigma, Y_{t}\right)$ and $X_{t}(x)=Y_{t}(x)$ for $t \leq 0$. Fix $w \in h^{-1}\left(X_{1}(x)\right)$. Then $h\left(Y_{t}(w)\right)=X_{\tau(w, t)}\left(X_{1}(x)\right)$ for all $t \in \mathbf{R}$ and $d(h(y), y)<\varepsilon$ for all $y \in M$. It is easy to see that the positive orbit $\left\{Y_{t}(w): t \geq 0\right\}$ is included in some small neighborhood $U(p)$ of $p$ since $X_{t}\left(X_{1}(x)\right) \rightarrow p$ as $0<t \nearrow \infty$.

On the other hand, take $t^{\prime}<0$ such that $X_{\tau\left(w, t^{\prime}\right)+1}(x) \in W_{r_{0} / 2}^{u}\left(\sigma, X_{t}\right)$. Then $Y_{t^{\prime}}(w) \in W_{r_{0}}^{u}\left(\sigma, X_{t}\right)=W_{r_{0}}^{u}\left(\sigma, Y_{t}\right)$ since $0<\varepsilon<r_{0} / 4$. By (1.3), every $Y_{t^{-}}$-orbit is actually a $X_{t}$-orbit if it is included in a set $B_{\varepsilon}\left(\left\{X_{t}(x): t \leq 0\right\}\right) \cap \overline{F_{x}\left(X_{t}, r_{0}, 1\right)^{c}}$. Thus, from (1.2), we can see that the $Y_{t^{-}}$orbit of $w$ passes through $V_{r^{\prime}}^{u}(x) \subset \Pi_{x, r_{0}}$ by the choice of $r^{\prime}>0$ since $0<\varepsilon<r^{\prime}$. Indeed, since

$$
Y_{t^{\prime}}(w) \in B_{\varepsilon}\left(\left\{X_{t}(x): t \leq 0\right\}\right) \cap \overline{F_{x}\left(X_{t}, r_{0}, 1\right)^{c}},
$$

we have $Y_{t}\left(Y_{t^{\prime}}(w)\right)=X_{t}\left(Y_{t^{\prime}}(w)\right)$ while

$$
Y_{t}\left(Y_{t^{\prime}}(w)\right) \in B_{\varepsilon}\left(\left\{X_{t}(x): t \leq 0\right\}\right) \cap \overline{F_{x}\left(X_{t}, r_{0}, 1\right)^{c}}
$$


by (1.3). If we fix the minimum number $t^{\prime \prime}>0$ such that $Y_{t^{\prime}+t^{\prime \prime}}(w) \in \Pi_{x, r_{0}}$, then

$$
Y_{t+t^{\prime}}(w)=Y_{t}\left(Y_{t^{\prime}}(w)\right) \in B_{\varepsilon}\left(\left\{X_{t}(x): t \leq 0\right\}\right) \cap \overline{F_{x}\left(X_{t}, r_{0}, 1\right)^{c}}
$$

for $0 \leq t \leq t^{\prime \prime}$. Thus $Y_{t^{\prime}+t^{\prime \prime}}(w)=X_{t^{\prime \prime}}\left(Y_{t^{\prime}}(w)\right) \in V_{r^{\prime}}^{u}(x)$ by $(1.2)$ since $Y_{t^{\prime}}(w) \in$ $W_{r_{0}}^{u}\left(\sigma, X_{t}\right)$. Hence, the orbit of $w$ also passes through $g\left(V_{r^{\prime}}^{u}(x)\right) \subset \Pi_{X_{1}(x)}$ (see Figure 1). Therefore, by the hyperbolicity of $p$, there is $\tilde{t} \geq 0$ such that $Y_{\tilde{t}}(w)$ escapes from $U(p)$ by (1.1). This is a contradiction and thus the proposition is proved in Case 1.

In Case 2, take a $C^{1}$ neighborhood $\mathcal{U}(X) \subset \mathcal{T} \mathcal{S}(M)$ of $X$ and set

$$
\begin{aligned}
& V^{s}\left(X_{-1}(x)\right)=\text { the connected component of } W^{s}\left(p, X_{t}\right) \cap \Pi_{X_{-1}(x)}, \\
& V^{u}\left(X_{-1}(x)\right)=\text { the connected component of } W^{u}\left(\sigma, X_{t}\right) \cap \Pi_{X_{-1}(x)}
\end{aligned}
$$

containing $X_{-1}(x)$ respectively. Let $f^{\prime}: \Pi_{X_{-1}(x), r_{0}} \rightarrow \Pi_{x}$ be as before, and, at first, we apply Lemma 1.2 to $X$ and $f^{\prime}$. For the above $\mathcal{U}(X)$, let $\varepsilon^{\prime}=\varepsilon^{\prime}(\mathcal{U}(X))>$ 0 be given by Lemma 1.2. Since $\hat{\Pi}_{x} \neq T_{x} V^{s}(x)+T_{x} V^{u}(x)$ and $\operatorname{dim} V^{s}(x)+$ $\operatorname{dim} V^{u}(x) \geq \operatorname{dim} \Pi_{x}$, there are $0<r_{1}<r_{0} / 4, \tilde{\varphi} \in \mathcal{N}_{\varepsilon^{\prime}}\left(\Pi_{X_{-1}(x), r_{0}}\right)$ and a submanifold $V\left(X_{-1}(x)\right) \subset \Pi_{X_{-1}(x), r_{0}}$ such that

- $V^{s}\left(X_{-1}(x)\right) \cap B_{r_{1}}\left(X_{-1}(x)\right) \subset V\left(X_{-1}(x)\right)$,

- $\tilde{\varphi}\left(V^{u}\left(X_{-1}(x)\right) \cap B_{r_{1}}\left(X_{-1}(x)\right)\right) \subset V\left(X_{-1}(x)\right)$ and $\tilde{\varphi}\left(X_{-1}(x)\right)=X_{-1}(x)$, 2).

- $\operatorname{dim} V^{s}(x)+\operatorname{dim} V^{u}(x)-\operatorname{dim} \Pi_{x}<\operatorname{dim} V\left(X_{-1}(x)\right)<\operatorname{dim} \Pi_{X_{-1}(x)}$ (see Figure

Let $Y \in \mathcal{U}(X)$ and $g=f^{\prime} \circ \tilde{\varphi}: \Pi_{X_{-1}(x), r_{0}} \rightarrow \Pi_{x}$ (since $g\left(X_{-1}(x)\right)=x$ ) be given by Lemma 1.2 and set

$$
\begin{aligned}
& V^{s}\left(x, Y_{t}\right)=\text { the connected component of } W^{s}\left(p, Y_{t}\right) \cap \Pi_{x} \text { containing } x, \\
& V^{u}\left(x, Y_{t}\right)=\text { the connected component of } W^{u}\left(\sigma, Y_{t}\right) \cap \Pi_{x} \text { containing } x .
\end{aligned}
$$

If we put $V\left(x, Y_{t}\right)=f^{\prime}\left(V\left(X_{-1}(x)\right)\right)$, then $\operatorname{dim} V\left(x, Y_{t}\right)<\operatorname{dim} \Pi_{x}$ and it is easy to see that there is $0<r_{2}<r_{0} / 4$ satisfying

$$
V^{u}\left(x, Y_{t}\right) \cap B_{r_{2}}(x) \subset V\left(x, Y_{t}\right) \text { and } V^{s}\left(x, Y_{t}\right) \cap B_{r_{2}}(x) \subset V\left(x, Y_{t}\right) .
$$

Note that by the choice of $r_{0}$, the map $f: \Pi_{x, r_{0}} \rightarrow \Pi_{X_{1}(x)}$ is also a Poincaré map for $Y_{t}, \sigma \in \operatorname{Sing}(Y) \cup P O\left(Y_{t}\right), X_{t}(x)=Y_{t}(x)$ for $t \leq 0$, and $W_{2 r_{0}}^{u}\left(\sigma, X_{t}\right)=W_{2 r_{0}}^{u}\left(\sigma, Y_{t}\right)$ since $Y(y)=X(y)$ if $y \notin F_{X_{-1}(x)}\left(X_{t}, r_{0}, 1\right)$.

Next, we make use of Lemma 1.2 for $Y$ and $f$. For $\delta>0$, there exists $\varepsilon(\delta)>0$ such that if $\varphi \in \mathcal{N}_{\varepsilon(\delta)}\left(\Pi_{x, r_{0}}\right)$, then the corresponding vector field $Z$ given by Lemma 1.2 satisfies $d_{C^{0}}(Y, Z)<\delta$. Put

$$
V_{r}^{u}\left(x, Y_{t}\right)=\text { the connected component of } V^{u}\left(x, Y_{t}\right) \cap B_{r}(x) \text { containing } x
$$

for $r>0$. Since $\operatorname{dim} V\left(x, Y_{t}\right)<\operatorname{dim} \Pi_{x}$, the following is easily obtained: there is $0<r^{\prime}<r_{2}$ with the property that

$(2.1)$ for every $\delta>0$, there exists $\varphi_{\delta} \in \mathcal{N}_{\varepsilon(\delta)}\left(\Pi_{x, r_{0}}\right)$ satisfying $\varphi_{\delta}\left(V_{r^{\prime}}^{u}\left(x, Y_{t}\right)\right) \cap$ $V\left(x, Y_{t}\right)=\emptyset$,

(2.2) $V_{r^{\prime}}^{u}\left(x, Y_{t}\right)=\left[\bigcup_{0 \leq t \leq t_{1}} Y_{t}\left(W_{2 r_{0}}^{u}\left(\sigma, Y_{t}\right)\right)\right] \cap \Pi_{x, r_{0}} \cap B_{r^{\prime}}(x) \quad$ (see Figure 3),

(2.3) $B_{r^{\prime}}\left(\left\{Y_{t}(x): t \leq 0\right\}\right) \cap \Pi_{x, r_{0}}=B_{r^{\prime}}(x) \cap \Pi_{x, r_{0}}$.

Here $t_{1}>0$ is a number such that $Y_{-t_{1}}(x) \in W_{r_{0}}^{u}\left(\sigma, Y_{t}\right)$. Thus $\varphi_{\delta}\left(V_{r^{\prime}}^{u}\left(x, Y_{t}\right)\right) \cap$ $V^{s}\left(x, Y_{t}\right)=\emptyset$ by $(2.1)$. Since $Y$ is topologically stable, we can apply the method used in the proof of the first case to $Y$. For a sufficiently small $0<\varepsilon<r^{\prime}$, pick 


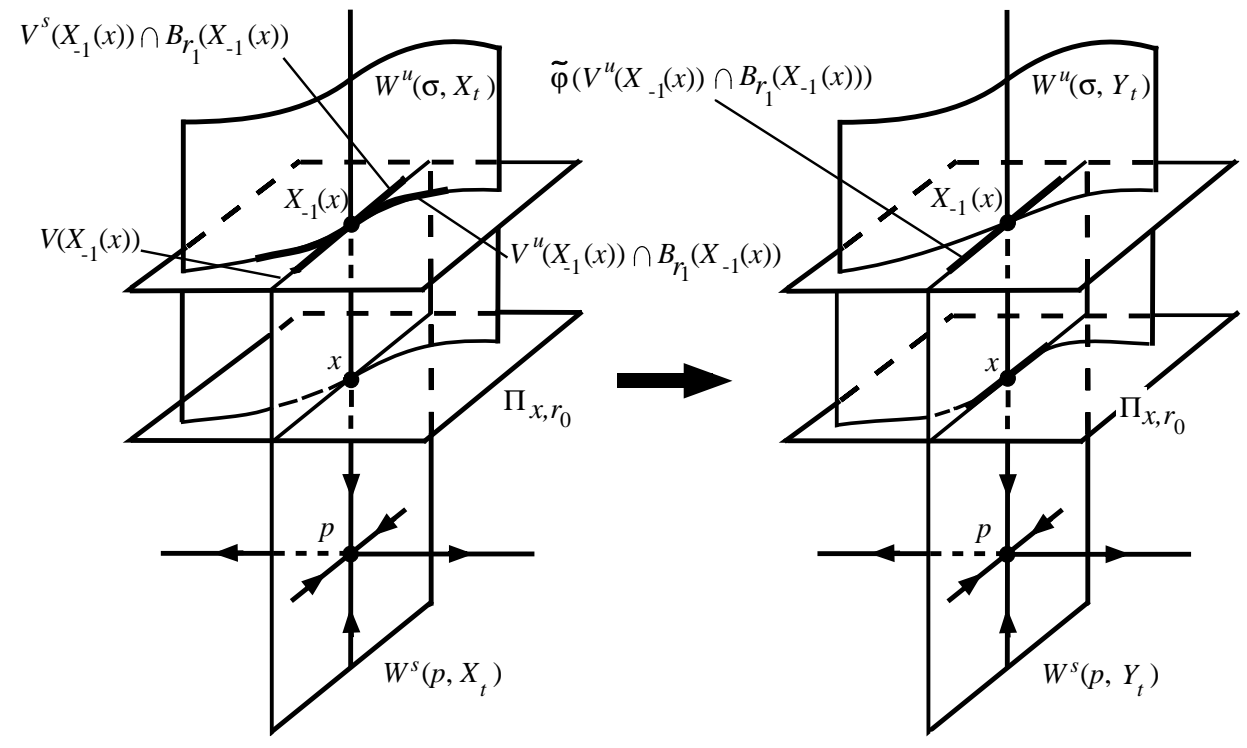

FIGURE 2.

$0<\delta<\varepsilon$ as in the definition of the topological stability of $Y_{t}$. Let $Z^{\delta}$ be the vector field given by Lemma 1.2 for the above perturbation (2.1); that is,

$$
\begin{cases}Z^{\delta}(y)=Y(y) & \text { if } y \notin F_{x}\left(Y_{t}, r_{0}, 1\right), \\ g^{\prime}(y)=f \circ \varphi_{\delta}(y) & \text { if } y \in \Pi_{x, r_{0}}, \\ d_{C^{0}}\left(Y, Z^{\delta}\right)<\delta, & \end{cases}
$$

where $g^{\prime}: \Pi_{x, r_{0}} \rightarrow \Pi_{Y_{1}(x)}$ is the Poincaré map induced by $Z_{t}^{\delta}$. For simplicity, denote $Z^{\delta}$ by $Z$.

Then, since $d_{C^{0}}(Y, Z)<\delta$, there are $h: M \rightarrow M$ and $\tau: M \times \mathbf{R} \rightarrow \mathbf{R}$ such that $h\left(Z_{t}(y)\right)=Y_{\tau(y, t)}(h(y))$ for all $y \in M$ and $d(h, i d)<\varepsilon$. Remark that

$$
\left(\left\{Y_{t}(x): t<0\right\} \cup W_{2 r_{0}}^{u}\left(\sigma, Y_{t}\right)\right) \cap \Pi_{x, r_{0}}=\emptyset
$$

and $Z(y)=Y(y)$ if $y \notin F_{x}\left(Y_{t}, r_{0}, 1\right)$. Thus $\sigma$ is also a hyperbolic singularity or periodic orbit of $Z$. Fix $w \in h^{-1}\left(Y_{1}(x)\right)$. Then $h\left(Z_{t}(w)\right)=Y_{\tau(w, t)}\left(Y_{1}(x)\right)$ for all $t \in \mathbf{R}$ and $d(h(y), y)<\varepsilon$ for all $y \in M$. It is easy to see that the positive orbit $\left\{Z_{t}(w): t \geq 0\right\}$ is included in some small neighborhood $U(p)$ of $p$ since $Y_{t}\left(Y_{1}(x)\right) \rightarrow p$ as $0<t \nearrow \infty$.

As in the first case, there exists $t^{\prime}<0$ such that $Z_{t^{\prime}}(w) \in W_{r_{0}}^{u}\left(\sigma, Z_{t}\right)=W_{r_{0}}^{u}\left(\sigma, Y_{t}\right)$ because $\varepsilon$ is sufficiently small. By $(2.3)$, every $Z_{t}$-orbit is actually a $Y_{t}$-orbit while it is included in $B_{\varepsilon}\left(\left\{Y_{t}(x): t \leq 0\right\}\right) \cap \overline{F_{x}\left(Y_{t}, r_{0}, 1\right)^{c}}$. Thus, we can check that the $Z_{t}$-orbit of $w$ passes through $V_{r^{\prime}}^{u}\left(x, Y_{t}\right)$ by the choice of $r^{\prime}>0$ since $0<\varepsilon<r^{\prime}$. Indeed, since

$$
Z_{t^{\prime}}(w) \in B_{\varepsilon}\left(\left\{Y_{t}(x): t \leq 0\right\}\right) \cap \overline{F_{x}\left(Y_{t}, r_{0}, 1\right)^{c}},
$$

if we pick the smallest number $t^{\prime \prime}>0$ such that $Z_{t^{\prime}+t^{\prime \prime}}(w) \in \Pi_{x, r_{0}}$, then $Z_{t^{\prime}+t^{\prime \prime}}(w)=$ $Z_{t^{\prime \prime}}\left(Z_{t^{\prime}}(w)\right)=Y_{t^{\prime \prime}}\left(Z_{t^{\prime}}(w)\right) \in V_{r^{\prime}}^{u}\left(x, Y_{t}\right)$ by (2.2). Hence the orbit of $w$ also passes through $g^{\prime}\left(V_{r^{\prime}}^{u}\left(x, Y_{t}\right)\right)$ (see Figure 3 ). Therefore, by the hyperbolicity of $p$, there is 


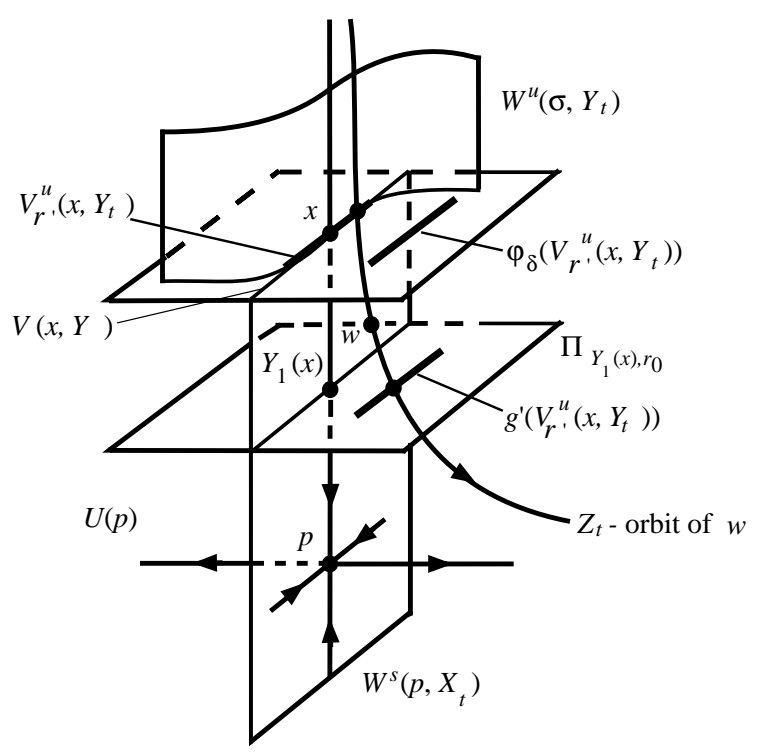

FIGURE 3.

$\tilde{t} \geq 0$ such that $Z_{\tilde{t}}(w)$ escapes from $U(p)$ because $\varphi_{\delta}\left(V_{r^{\prime}}^{u}\left(x, Y_{t}\right)\right) \cap V^{s}\left(x, Y_{t}\right)=\emptyset$. This is contradiction and the proof is complete.

\section{Proof of Proposition C}

Let $\mathcal{T S}(M)$ be as before and fix $X \in \operatorname{int} \mathcal{T} \mathcal{S}(M)$. Suppose that $\gamma, \gamma^{\prime} \in P O\left(X_{t}\right)$ are hyperbolic and $x \in W^{s}\left(\gamma, X_{t}\right) \cap W^{u}\left(\gamma^{\prime}, X_{t}\right)$. We can prove the transversality of $\gamma$ and $\gamma^{\prime}$ (at $x$ ) independently of the sum $\operatorname{dim} W^{s}\left(\gamma, X_{t}\right)+\operatorname{dim} W^{u}\left(\gamma^{\prime}, X_{t}\right)$ (cf. section 3). Basically, however, an idea of the proof is the same as that of Proposition B, and the same argument for diffeomorphisms was displayed in [13, Proof of Proposition B].

Fix $p \in \gamma\left(X_{T}(p)=p, T>0\right)$ and let $r_{0}>0$ be sufficiently small so that we can define the Poincaré map $f: \Pi_{p, r_{0}} \rightarrow \Pi_{p}$. Since $p$ is hyperbolic, there are a $D f$-invariant splitting $\hat{\Pi}_{p}=E_{p}^{s} \oplus E_{p}^{u}$ and two constants $C>0,0<\lambda<1$ such that

$$
\left\|D f_{\mid E_{p}^{s}}^{m}\right\|<C \lambda^{m} \text { and }\left\|D f_{\mid E_{p}^{u}}^{-m}\right\|<C \lambda^{m}
$$

for all $m \geq 0$. Throughout this section, let

$$
W_{r}^{\sigma}(p, f)=\text { the connected component of } W^{\sigma}\left(\gamma, X_{t}\right) \cap \Pi_{p, r} \text { containing } p
$$

for $\sigma=s, u$ and $0<r \leq r_{0}$.

Before starting the argument, we shall give a rough outline of our proof. Let

$$
x \in W^{s}(p, f) \cap\left(W^{u}\left(\gamma^{\prime}, X_{t}\right) \cap \Pi_{p}\right) .
$$

First of all, we linearize $f$ at $p$ (with respect to the exponential coordinate by using Lemma 1.3) with a small perturbation without changing the above two sets near $x$. Next, with a small modification of the linearized map, we arrange that there is a neighborhood of $x$ in $W^{u}\left(\gamma^{\prime}, X_{t}\right) \cap \Pi_{p}$ which arrives, through iteration of the map, in the linearized neighborhood of $p$ as a piece of an affine space (with respect to 
the exponential coordinates). If the intersection $W_{r_{0}}^{s}(p, f)$ and $W^{u}\left(\gamma^{\prime}, X_{t}\right) \cap \Pi_{p}$ is not transverse at $x$, then the piece of an affine space is not transverse with respect to the local linear stable manifold of $p$. The modified vector field $Y \in \mathcal{X}^{1}(M)$ is topologically stable because the above two modifications are sufficiently $C^{1}$ small. However, since the piece of an affine space is not transverse with respect to the local linear stable manifold of $p$, we can construct $Z \in \mathcal{X}^{1}(M)$ (near $Y$ with respect to the $C^{0}$ topology) and can take $w \in h^{-1}(x)$ whose positive $Z$-orbit escapes from a small tubular neighborhood of $\gamma$. Here $h$ is a semi-conjugacy from $Z$ to $Y$. This is a contradiction since $x \in W^{s}\left(\gamma, Y_{t}\right)$ and $d(h, i d)$ is sufficiently small.

Suppose that $x \in W_{r_{0} / 2}^{s}(p, f) \backslash i n t W_{r_{0} / 2}^{s}(p, f)$. The following is a simple generalization of Lemma 1.3. Let $T^{\prime}>0$ be the number with $f(x)=X_{T^{\prime}}(x)$ and take $0<r_{1}<r_{0} / 4$ such that $F_{p}\left(X_{t}, r_{1}, T\right) \cap F_{x}\left(X_{t}, r_{1}, T^{\prime}\right)=\emptyset$.

Lemma 4.1. Under the above notation, for every $C^{1}$ neighborhood $\mathcal{U}(X)$ of $X$, there are $0<\varepsilon_{0}<r_{0} / 4$ and $Y \in \mathcal{U}(X)$ satisfying

(i) $Y(y)=X(y)$ if $y \notin F_{p}\left(X_{t}, r_{1}, T\right) \cup F_{x}\left(X_{t}, r_{1}, T^{\prime}\right)$,

(ii) $\gamma, \gamma^{\prime} \in P O\left(Y_{t}\right)$ and $Y_{T}(p)=p \in \gamma$,

(iii) $g(y)= \begin{cases}\exp _{p} \circ D_{p} f \circ \exp _{p}^{-1}(y) & \text { if } y \in B_{\varepsilon_{0} / 4}(p) \cap \Pi_{p, r_{0}}, \\ f(y) & \text { if } y \notin B_{\varepsilon_{0}}(p) \cap \Pi_{p, r_{0}},\end{cases}$

(iv) $g(p)=p, x \in W_{r_{0}}^{s}(p, g)$ and $T_{x} W_{r_{0}}^{s}(p, g)=T_{x} W_{r_{0}}^{s}(p, f)$,

(v) $T_{x} W^{u}\left(\gamma^{\prime}, Y_{t}\right)=T_{x} W^{u}\left(\gamma^{\prime}, X_{t}\right)$.

Here $g: \Pi_{p, r_{0}} \rightarrow \Pi_{p}$ is the Poincaré map of $Y_{t}$ and $W_{r_{0}}^{\sigma}(p, g)$ is the connected component of $W^{\sigma}\left(\gamma, Y_{t}\right) \cap \Pi_{p, r_{0}}$ containing $p(\sigma=s, t)$.

Proof. Let $\mathcal{U}(X)$ be given. For every $C^{1}$ neighborhood $\mathcal{V}(X) \subset \mathcal{U}(X)$ of $X$, by Lemma 1.3, there are $0<\varepsilon_{0}<r_{0} / 4$ and $Z \in \mathcal{V}(X)$ such that

- $Z(y)=X(y)$ if $y \notin F_{p}\left(X_{t}, r_{1}, T\right)$,

- $Z_{T}(p)=p \in \gamma \in P O\left(Z_{t}\right)$,

- $g_{Z}(y)= \begin{cases}\exp _{p} \circ D_{p} f \circ \exp _{p}^{-1}(y) & \text { if } y \in B_{\varepsilon_{0} / 4}(p) \cap \Pi_{p, r_{1}}, \\ f(y) & \text { if } y \notin B_{\varepsilon_{0}}(p) \cap \Pi_{p, r_{1}},\end{cases}$

where $g_{Z}: \Pi_{p, r_{1}} \rightarrow \Pi_{p}$ is the Poincaré map of $Z_{t}$. Extend $g_{Z}$ to the whole space $\Pi_{p, r_{0}}$ by the formula $g_{Z}(y)=f(y)$ if $y \in \Pi_{p, r_{0}} \backslash \Pi_{p, r_{1}}$. Then $g_{Z} \rightarrow f$ with respect to the $C^{1}$ topology as $Z \rightarrow X$, and thus $W_{r_{0}}^{s}\left(p, g_{Z}\right) \rightarrow W_{r_{0}}^{s}(p, f)$ with respect to the $C^{1}$ topology as $Z \rightarrow X$. Hence, for every $\varepsilon>0$, we find $Z \in \mathcal{U}(X), 0<r_{2}<r_{1} / 2$ and $\varphi \in \mathcal{N}_{\varepsilon}\left(\Pi_{x, 2 r_{2}}\right)\left(\Pi_{x, 2 r_{2}} \subset \Pi_{p, r_{0}}\right)$ such that

- $\varphi$ embeds $W_{r_{0}}^{s}(p, f) \cap B_{r_{2} / 4}(x)$ into $W_{r_{0}}^{s}\left(p, g_{Z}\right)$; that is,

$$
\varphi\left(W_{r_{0}}^{s}(p, f) \cap B_{r_{2} / 4}(x)\right) \subset W_{r_{0}}^{s}\left(p, g_{Z}\right),
$$

- $\varphi(y)=y$ if $y \notin \Pi_{x, r_{2}}$,

Now we apply Lemma 1.2 to $Z$ and $g_{Z \mid \Pi_{p, r_{0}} \cap B_{2 r_{2}}(x)}$. Since $\varepsilon$ is arbitrary, by Lemma 1.2 , there is $Y \in \mathcal{U}(X)$ (sufficiently near $Z$ ) satisfying

$$
\begin{cases}Y(y)=Z(y) & \text { if } y \notin F_{x}\left(Z_{t}, 2 r_{2}, T^{\prime}\right), \\ g(y)=g_{Z} \circ \varphi(y) & \text { if } y \in \Pi_{x, 2 r_{2}},\end{cases}
$$

where $g: \Pi_{x, 2 r_{2}} \rightarrow \Pi_{Z_{T^{\prime}}(x)}$ is the Poincaré map defined by $Y_{t}$. If we extend $g$ to $\Pi_{p, r_{0}}$ by $g(y)=g_{Z}(y)$ when $y \in \Pi_{p, r_{0}} \backslash \Pi_{x, 2 r_{2}}$, then $g(p)=p, x \in W_{r_{0}}^{s}(p, g)$ and $T_{x} W_{r_{0}}^{s}(p, g)=T_{x} W_{r_{0}}^{s}(p, f)$ by $(\dagger)$. It is easy to see that there is $0<r^{\prime}<r_{1}$ such that

$$
X_{-t}\left(C_{r^{\prime}}^{u}(x)\right) \cap\left(F_{p}\left(X_{t}, r_{1}, T / 2\right) \cup F_{x}\left(Z_{t}, 2 r_{2}, T^{\prime}\right)\right)=\emptyset
$$


for all $t>0$. Here $C_{r^{\prime}}^{u}(x)$ is the connected component of $W^{u}\left(\gamma^{\prime}, X_{t}\right) \cap \Pi_{p} \cap B_{r^{\prime}}(x)$ containing $x$. Thus

$$
T_{x} W^{u}\left(\gamma^{\prime}, Y_{t}\right)=T_{x} W^{u}\left(\gamma^{\prime}, Z_{t}\right)=T_{x} W^{u}\left(\gamma^{\prime}, X_{t}\right) .
$$

The lemma is proved.

Put $E_{x}^{\sigma}(\varepsilon)=\left\{v \in E_{x}^{\sigma} \mid\|v\| \leq \varepsilon\right\}$ for $\varepsilon>0(\sigma=s, u)$, and let $g \in E m b^{1}\left(\Pi_{p, r_{0}}, \Pi_{p}\right)$, $p=g(p) \in \Pi_{p}$ and $\varepsilon_{0}>0$ be given by Lemma 4.1. Then it is easily checked that

$$
\exp _{p}\left(E_{p}^{\sigma}\left(\varepsilon_{0} / 4\right)\right) \subset W_{r_{0}}^{\sigma}(p, g) \text { and } \operatorname{dim} \exp _{p}\left(E_{p}^{\sigma}\left(\varepsilon_{0} / 4\right)\right)=\operatorname{dim} W_{r_{0}}^{\sigma}(p, g)
$$

for $\sigma=s, u$ since $\varepsilon_{0}$ is small. For convenience, we denote $\exp _{p}\left(E_{p}^{\sigma}(\varepsilon)\right)$ by $W_{\varepsilon}^{\sigma}(p, g)$ for $\sigma=s, u$ and for $0<\varepsilon \leq \varepsilon_{0} / 4$.

Proof of Proposition C. Let $X \in \operatorname{int} \mathcal{T} \mathcal{S}(M)$. Suppose that $\gamma, \gamma^{\prime} \in P O\left(X_{t}\right)$ are hyperbolic and $x \in W^{s}\left(\gamma, X_{t}\right) \cap W^{u}\left(\gamma^{\prime}, X_{t}\right)$. Let $p \in \gamma\left(X_{T}(p)=p, T>0\right)$ and $f: \Pi_{p, r_{0}} \rightarrow \Pi_{p}\left(r_{0}>0\right)$ be as before. We may assume that

- $x \in W_{r_{0} / 2}^{s}(p, f) \backslash i n t W_{r_{0} / 2}^{s}(p, f)$,

- $W_{2 r_{0}}^{u}\left(\gamma^{\prime}, X_{t}\right) \cap \Pi_{p, r_{0}}=\emptyset$.

Here $W_{r_{0}}^{u}\left(\gamma^{\prime}, X_{t}\right)$ is the local unstable manifold of $\gamma^{\prime}$. Let $T^{\prime}>0,0<r_{1}<r_{0} / 4$, $F_{p}\left(X_{t}, r_{1}, T\right)$ and $F_{x}\left(X_{t}, r_{1}, T^{\prime}\right)$ be chosen in the next paragraph of the rough outline of the proof. We may assume further that

- $\left\{X_{t}(x): t<0\right\} \cap\left(F_{p}\left(X_{t}, r_{1}, T\right) \cup F_{x}\left(X_{t}, r_{1}, T^{\prime}\right)\right)=\emptyset$.

Fix a $C^{1}$ neighborhood $\mathcal{U}(X) \subset \mathcal{T} \mathcal{S}(M)$ of $X$, and let $0<\varepsilon_{0}<r_{0} / 4, Y \in \mathcal{U}(X)$ and $g$ be given by Lemma 4.1. Thus

$$
T_{x} W_{r_{0}}^{s}(p, g)=T_{x} W_{r_{0}}^{s}(p, f), W_{2 r_{0}}^{u}\left(\gamma^{\prime}, X_{t}\right)=W_{2 r_{0}}^{u}\left(\gamma^{\prime}, Y_{t}\right)
$$

and $X_{t}(x)=Y_{t}(x)$ for $t \leq 0$. Clearly,

$$
1 \leq \operatorname{dim} W_{r_{0}}^{s}(p, g) \leq \operatorname{dim} \Pi_{p} \text { and } 1 \leq \operatorname{dim}\left(W^{u}\left(\gamma^{\prime}, Y_{t}\right) \cap \Pi_{p}\right) \leq \operatorname{dim} \Pi_{p} .
$$

If $\operatorname{dim} W_{r_{0}}^{s}(p, g)=\operatorname{dim} \Pi_{p}$ or $\operatorname{dim}\left(W^{u}\left(\gamma^{\prime}, Y_{t}\right) \cap \Pi_{p}\right)=\operatorname{dim} \Pi_{p}$, then the conclusion is clear.

Pick $\ell>0$ so large that $g^{\ell-1}(x) \in W_{\varepsilon_{0} / 8}^{s}(p, g)$, and set

$$
C^{u}\left(g^{\ell}(x)\right)=\text { the connected component of } W^{u}\left(\gamma^{\prime}, Y_{t}\right) \cap \Pi_{p} \text { containing } g^{\ell}(x) \text {. }
$$

Hereafter, to simplify notation, denote $g^{\ell}(x)$ by $x$ (see Figure 4 ).

Then

$$
\exp _{p}^{-1}\left(C^{u}(x)\right) \subset \hat{\Pi}_{p} \text { and } T_{x} C^{u}(x)=T_{x}\left(W^{u}\left(\gamma^{\prime}, Y_{t}\right) \cap \Pi_{p}\right) .
$$

For a linear subspace $E$ of $\hat{\Pi}_{p}$ and $\nu>0$, let

$$
E_{\nu}(x)=\left\{v+\exp _{p}^{-1}(x) \mid v \in E \text { with }\|v\| \leq \nu\right\}
$$

be a piece of an affine space running parallel to $E$.

Let $T^{\prime \prime}, T^{\prime \prime \prime}>0$ be numbers with $Y_{T^{\prime \prime}}\left(g^{-1}(x)\right)=x, Y_{T^{\prime \prime \prime}}(x)=g(x)$ respectively. Choose a linear subspace $E^{\prime} \subset \hat{\Pi}_{p}$ and $0<\nu_{0} \leq \varepsilon_{0} / 8$ such that

- for every $0<\nu \leq \nu_{0}, \exp _{p}\left(E_{\nu}^{\prime}(x)\right) \subset B_{\varepsilon_{0} / 4}(p)$,

$$
\begin{aligned}
& \text { (**) } \quad T_{x} \exp _{p}\left(E_{\nu_{0}}^{\prime}(x)\right)=T_{x} C^{u}(x), \\
& \text { - }\left(F_{g^{-1}(x)}\left(Y_{t}, \nu_{0}, T^{\prime \prime}\right) \cup F_{x}\left(Y_{t}, \nu_{0}, T^{\prime \prime \prime}\right)\right) \cap \gamma=\emptyset \text {, } \\
& \text { - }\left\{Y_{t}(x): t<-1\right\} \cap F_{g^{-1}(x)}\left(Y_{t}, \nu_{0}, T^{\prime \prime}\right)=\emptyset \text { and }\left\{Y_{t}(x): t<0\right\} \cap F_{x}\left(Y_{t}, \nu_{0}, T^{\prime \prime \prime}\right) \\
& =\emptyset \text {, }
\end{aligned}
$$




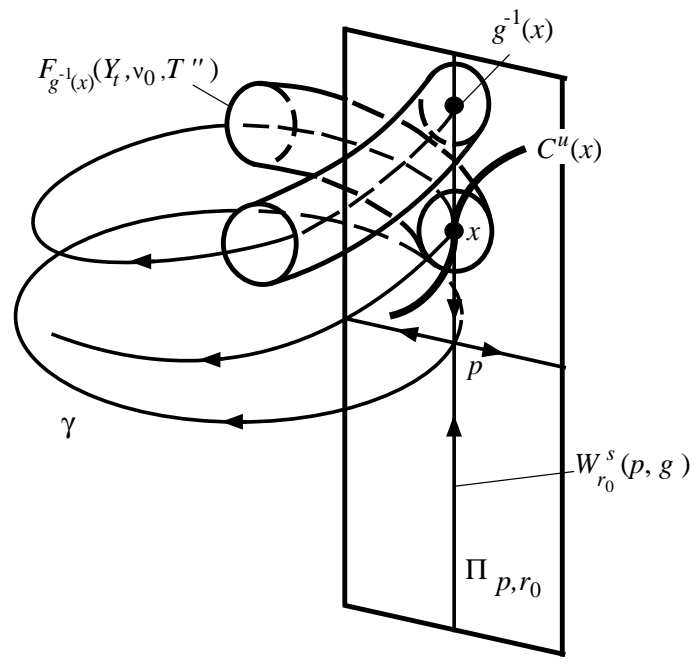

FIGURE 4.

$$
\begin{gathered}
B_{\nu_{0}}\left(\left\{Y_{t}(x): t \leq 0\right\}\right) \cap F_{x}\left(Y_{t}, \nu_{0}, T^{\prime \prime \prime}\right) \cap \Pi_{p, r_{0}} \\
=B_{\nu_{0}}(x) \cap F_{x}\left(Y_{t}, \nu_{0}, T^{\prime \prime \prime}\right) \cap \Pi_{p, r_{0}},
\end{gathered}
$$

- $g^{i}\left(W_{r_{0}}^{s}(p, g) \cap B_{\nu_{0}}\left(g^{-1}(x)\right)\right) \cap B_{\nu_{0}}\left(g^{-1}(x)\right)=\emptyset$ for $i \geq 1$,

- $Y_{-t}\left(C^{u}\left(g^{-1}(x)\right)\right) \cap B_{\nu_{0}}\left(g^{-1}(x)\right)=\emptyset$ for $t>0$.

Here $C^{u}\left(g^{-1}(x)\right)$ is the connected component of $W^{u}\left(\gamma^{\prime}, Y_{t}\right) \cap \Pi_{p} \cap B_{\nu_{0}}\left(g^{-1}(x)\right)$ containing $g^{-1}(x)$. The above properties on $F_{x}\left(Y_{t}, \nu_{0}, T^{\prime \prime \prime}\right)$ will be used later in the proof of Lemma 4.3.

As in the proof of Lemma 4.1, we have the following lemma (apply Lemma 1.2 to $Y$ and $\left.g_{\mid \Pi_{p, r_{0}} \cap B_{\nu_{0}}\left(g^{-1}(x)\right)}\right)$.

Lemma 4.2. Fix a $C^{1}$ neighborhood $\mathcal{U}(Y) \subset \mathcal{U}(X)$ of $Y$. Then there are $0<\nu_{1}<$ $\nu_{0} / 4$ and $Y^{\prime} \in \mathcal{U}(Y)$ such that

(i) $Y^{\prime}(y)=Y(y)$ if $y \notin F_{g^{-1}(x)}\left(Y_{t}, \nu_{0}, T^{\prime \prime}\right)$,

(ii) $Y_{T^{\prime \prime}}^{\prime}\left(g^{-1}(x)\right)=x$,

(iii) $\exp _{p}\left(E_{\nu_{1}}^{\prime}(x)\right) \subset W^{u}\left(\gamma^{\prime}, Y_{t}^{\prime}\right) \cap \Pi_{p}$ and

$$
T_{x} \exp _{p}\left(E_{\nu_{1}}^{\prime}(x)\right)=T_{x}\left(W^{u}\left(\gamma^{\prime}, Y_{t}^{\prime}\right) \cap \Pi_{p}\right)
$$

(see Figure 5).

Remark 4.1. (i) We see that $W_{2 r_{0}}^{u}\left(\gamma^{\prime}, Y\right)=W_{2 r_{0}}^{u}\left(\gamma^{\prime}, Y^{\prime}\right)$ and $Y_{t}(x)=Y_{t}^{\prime}(x)$ for $t \leq 0$

(ii) Let $g^{\prime}: \Pi_{p, r_{0}} \rightarrow \Pi_{p}$ be the Poincaré map induced by $Y_{t}^{\prime}$. Then, by Lemma $4.2(i), \gamma^{\prime} \in P O\left(Y_{t}^{\prime}\right)$ and $g^{\prime}(y)=g(y)$ if $y \in \Pi_{p, r_{0}} \backslash B_{\nu_{0}}\left(g^{-1}(x)\right)$. Thus $g^{\prime}(p)=g(p)$. By Lemma $4.2(i i), g^{\prime i}(x)=g^{i}(x)$ for all $i \geq 0$.

(iii) By the perturbation used in the above lemma, $W_{\varepsilon_{0} / 4}^{s}(p, g)$ may be deformed near $g^{-j}(x)$ if $g^{-j}(x) \in W_{\varepsilon_{0} / 4}^{s}(p, g)$ for some $j>0$. We denote the deformed manifold by $W_{\varepsilon_{0} / 4}^{s}\left(p, g^{\prime}\right)$ (see Figure 5). Then $x \in W_{\varepsilon_{0} / 4}^{s}\left(p, g^{\prime}\right)$ and

$$
T_{x} W_{\varepsilon_{0} / 4}^{s}\left(p, g^{\prime}\right)=T_{x} W_{\varepsilon_{0} / 4}^{s}(p, g) .
$$




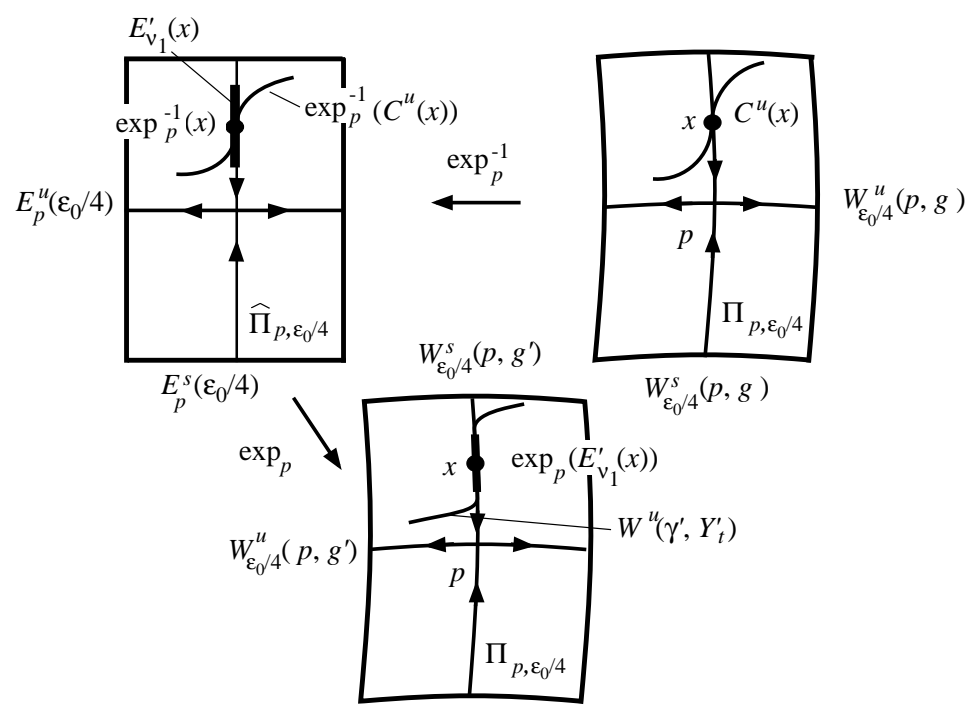

FiguRE 5.

Remark 4.2. If $\exp _{p}\left(E_{\nu_{1}}^{\prime}(x)\right)$ does not meet $W_{\varepsilon_{0} / 4}^{s}\left(p, g^{\prime}\right)$ transversely at $x$, then the piece $E_{\nu_{1}}^{\prime}(x)$ of the affine space is not transverse with respect to the local linear stable manifold $E_{p}^{s}\left(\varepsilon_{0} / 4\right)$ of $p$ (see Figure 5$)$. Thus we can destroy the intersection "locally" with a small perturbation (see Figure 6).

Lemma 4.3. Under the above notation, $\exp _{p}\left(E_{\nu_{1}}^{\prime}(x)\right)$ meets $W_{\varepsilon_{0} / 4}^{s}\left(p, g^{\prime}\right)$ transversely at $x$.

If the conclusion of this lemma is established, then

$$
\hat{\Pi}_{p}=T_{x} W^{s}(x, f)+T_{x}\left(W^{u}\left(\gamma^{\prime}, X_{t}\right) \cap \Pi_{p}\right)
$$

since

$$
T_{x} W^{s}\left(x, g^{\prime}\right)=T_{x} W^{s}(x, g)=T_{x} W^{s}(x, f)
$$

and

$$
T_{x}\left(W^{u}\left(\gamma^{\prime}, X_{t}\right) \cap \Pi_{p}\right)=T_{x} \exp _{p}\left(E_{\nu_{1}}^{\prime}(x)\right)
$$

by $(*),(* *)$, Lemma $4.1(v)$, Lemma $4.2(i i i)$, and Remark $4.1(i i i)$. Therefore the proof of Proposition $\mathrm{C}$ is complete.

Proof of Lemma 4.3. To simplify notation, denote $Y^{\prime}, g^{\prime}$ and $W_{\varepsilon_{0} / 4}^{s}\left(p, g^{\prime}\right)$ by $Y, g$ and $W_{\varepsilon_{0} / 4}^{s}(p)$ etc., respectively.

We make use of Lemma 1.2 for $Y$ and $g_{\mid \Pi_{p, r_{0}} \cap B_{2 \nu_{1}}(x)}$. For $\delta>0$, there is $\varepsilon(\delta)>0$ such that if $\varphi \in \mathcal{N}_{\varepsilon(\delta)}\left(\Pi_{x, 2 \nu_{1}}\right)$, then the corresponding vector field $Z$ given by Lemma 1.2 satisfies $d_{C^{0}}(Y, Z)<\delta$. Let $t_{2}>0$ be a number such that $Y_{-t_{2}}(x) \in W_{r_{0}}^{u}\left(\gamma^{\prime}, Y_{t}\right)$. If $\exp _{p}\left(E_{\nu_{1}}^{\prime}(x)\right)$ does not meet $W_{\varepsilon_{0} / 4}^{s}(p)$ transversely at $x$, then, by Remark 4.2 it is not hard to show the following: there is $0<r^{\prime}<\nu_{1} / 2$ such that 


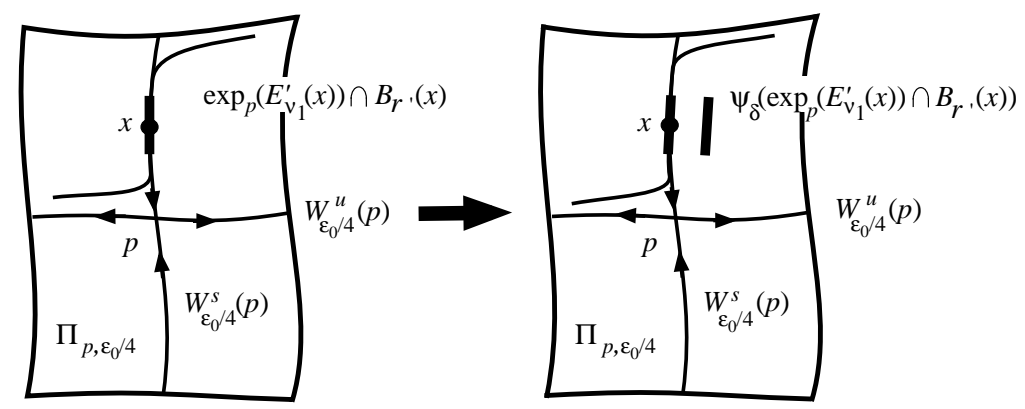

Figure 6.

(a) for every $\delta>0$, there is $\psi_{\delta} \in \mathcal{N}_{\varepsilon(\delta)}\left(\Pi_{x, 2 \nu_{1}}\right)$ satisfying

$$
\left\{\begin{array}{l}
\psi_{\delta}\left(\exp _{p}\left(E_{\nu_{1}}^{\prime}(x)\right) \cap B_{r^{\prime}}(x)\right) \cap W_{r_{0}}^{s}(p)=\emptyset \\
\psi_{\delta}(y)=y \text { if } y \notin \Pi_{x, \nu_{1}}
\end{array}\right.
$$

(b) $\exp _{p}\left(E_{\nu_{1}}^{\prime}(x)\right) \cap B_{r^{\prime}}(x)=\left[\bigcup_{0 \leq t \leq t_{2}} Y_{t}\left(W_{2 r_{0}}^{u}\left(\gamma^{\prime}, Y_{t}\right)\right)\right] \cap \Pi_{p, r_{0}} \cap B_{r^{\prime}}(x) \quad$ (see Figure 6).

Fix $0<\varepsilon<r^{\prime}$ sufficiently small and let $0<\delta<\varepsilon$ be as in the definition of the topological stability of $Y_{t}$. Let $Z^{\delta} \in \mathcal{X}^{1}(M)$ be given by Lemma 1.2 for the above perturbation (a); that is,

$$
\begin{cases}Z^{\delta}(z)=Y(z) & \text { if } z \notin F_{x}\left(Y_{t}, 2 \nu_{1}, T^{\prime \prime \prime}\right), \\ \tilde{g}(z)=g \circ \psi_{\delta}(z) & \text { if } z \in \Pi_{x, 2 \nu_{1}}, \\ d_{C^{0}}\left(Y, Z^{\delta}\right)<\delta . & \end{cases}
$$

Here $\tilde{g}: \Pi_{x, 2 \nu_{1}} \rightarrow \Pi_{g(x)}$ is the Poincaré map induced by $Z_{t}^{\delta}$.

By the choice of $\nu_{1}, F_{x}\left(Y_{t}, 2 \nu_{1}, T^{\prime \prime \prime}\right) \cap \gamma=\emptyset$ and so $Z_{t}^{\delta}(x)=Y_{t}(x)$ for $t \leq 0$. Extend $\tilde{g}$ to $\Pi_{p, r_{0}}$ by $\tilde{g}(z)=g(z)$ if $z \in \Pi_{p, r_{0}} \backslash \Pi_{x, 2 \nu_{1}}$, and denote $Z^{\delta}$ by $Z$ for simplicity.

Since $d_{C^{0}}(Y, Z)<\delta$, we can find $h: M \rightarrow M$ and $\tau: M \times \mathbf{R} \rightarrow \mathbf{R}$ such that $h\left(Z_{t}(z)\right)=Y_{\tau(z, t)}(h(z))$ for all $z \in M$ and $d(h, i d)<\varepsilon$. Remark that

$$
\left(\left\{Y_{t}(x): t<0\right\} \cup W_{2 r_{0}}^{u}\left(\gamma^{\prime}, Y_{t}\right)\right) \cap \Pi_{x, r_{0}}=\emptyset .
$$

Thus $\gamma^{\prime}$ is also a hyperbolic periodic orbit of $Z_{t}$ and $W_{2 r_{0}}^{u}\left(\gamma^{\prime}, Z_{t}\right)=W_{2 r_{0}}^{u}\left(\gamma^{\prime}, Y_{t}\right)$ since $Z(z)=Y(z)$ if $z \notin F_{x}\left(Y_{t}, 2 \nu_{1}, T^{\prime \prime \prime}\right)$. Fix $w \in h^{-1}(x)$. Then $h\left(Z_{t}(w)\right)=$ $Y_{\tau(w, t)}(x)$ for all $t \in \mathbf{R}$ and $d(h(z), z)<\varepsilon$ for all $z \in M$. As in the proof of Proposition $\mathrm{B}$, it is easy to see that there exists $t^{\prime}<0$ such that $Z_{t^{\prime}}(w) \in W_{r_{0}}^{u}\left(\gamma^{\prime}, Z_{t}\right)=$ $W_{r_{0}}^{u}\left(\gamma^{\prime}, Y_{t}\right)$ since $\varepsilon$ is sufficiently small. By $(\ddagger)$, every $Z_{t}$-orbit is actually a $Y_{t^{-}}$-orbit if it is included in $B_{\varepsilon}\left(\left\{Y_{t}(x): t \leq 0\right\}\right) \cap \overline{F_{x}\left(Y_{t}, 2 \nu_{1}, T^{\prime \prime \prime}\right)^{c}}$. Thus, by (b) we can see that the $Z_{t}$-orbit of $w$ passes through $\exp _{p}\left(E_{\nu_{1}}^{\prime}(x)\right) \cap B_{r^{\prime}}(x)$ by the choice of $r^{\prime}>0$ since $0<\varepsilon<r^{\prime}<\nu_{0}$. Indeed, since $Z_{t^{\prime}}(w) \in B_{\varepsilon}\left(\left\{Y_{t}(x): t \leq 0\right\}\right) \cap \overline{F_{x}\left(Y_{t}, 2 \nu_{1}, T^{\prime \prime \prime}\right)^{c}}$, there exists $t^{\prime \prime}>0$ such that $Z_{t^{\prime}+t^{\prime \prime}}(w) \in \Pi_{p, r_{0}}$ and

$$
Z_{t+t^{\prime}}(w) \in B_{\varepsilon}\left(\left\{Y_{t}(x): t \leq 0\right\}\right) \cap \overline{F_{x}\left(Y_{t}, 2 \nu_{1}, T^{\prime \prime \prime}\right)^{c}}
$$

for $0 \leq t \leq t^{\prime \prime}$. Thus $Z_{t^{\prime}+t^{\prime \prime}}(w)=Y_{t^{\prime \prime}}\left(Z_{t^{\prime}}(w)\right) \in \exp _{p}\left(E_{\nu_{1}}^{\prime}(x)\right) \cap B_{r^{\prime}}(x)$ by (b) since $Z_{t^{\prime}}(w) \in W_{r_{0}}^{u}\left(\gamma^{\prime}, Z_{t}\right)$, and hence, the orbit of $w$ also passes through

$$
g\left(\psi_{\delta}\left(\exp _{p}\left(E_{\nu_{1}}^{\prime}(x)\right) \cap B_{r^{\prime}}(x)\right)\right)
$$




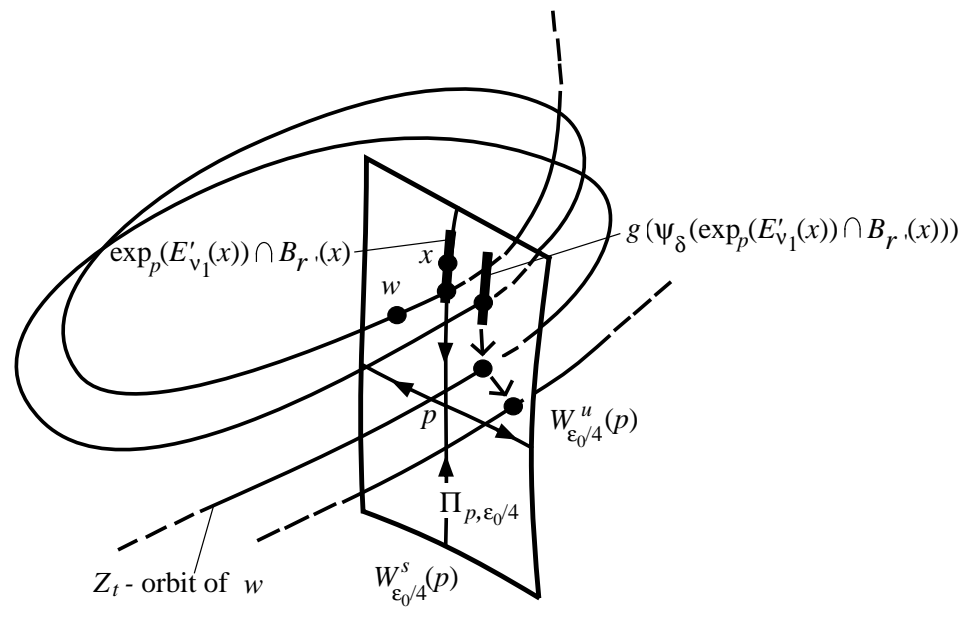

FIGURE 7.

(see Figure 7). Therefore, by (a) and the hyperbolicity of $\gamma$, there is $\tilde{t}>0$ such that $Z_{t}(w) \in B_{\varepsilon_{0} / 4}(\gamma)$ for all $0 \leq t<\tilde{t}$ and $Z_{\tilde{t}}(w) \notin B_{\varepsilon_{0} / 4}(\gamma)$ (see Figure 7 ). On the other hand, since $x \in W_{\varepsilon_{0} / 8}^{s}(p)$, we have $Z_{t}(w) \in B_{\varepsilon_{0} / 4}(\gamma)$ for all $t \geq 0$. This is a contradiction.

\section{ACKNOWLEDGEMENT}

We would like to thank Professor K. Hiraide for his useful comments and suggestions during the preparation of this paper.

\section{REFERENCES}

[1] P. Fleming and M. Hurley, A converse topological stability theorem for flows on surfaces, J. Diff. Eqs. 53 (1984), 172-191. MR 87j:58056

[2] J. Franks, Necessary conditions for stability of diffeomorphisms, Trans. Amer. Math. Soc. 158 (1971), 301-308. MR 44:1042

[3] S. Gan, Another proof for $C^{1}$ stability conjecture for flows, Sci. China Ser. A, 41 (1998), 1076-1082. MR 2000a:37006

[4] S. Hayashi, Connecting invariant manifolds and the solution of the $C^{1}$ stability and $\Omega$ stability conjectures for flows, Annals of Math. 145 (1997), 81-137; Correction, Annals of Math. 150 (1999), 353-356. MR 98b:58096, MR 2000h:37029

[5] M. Hurley, Consequences of topological stability, J. Diff. Eqs. 54 (1984), 60-72. MR 85m:58105

[6] M. Hurley, Bistable vector fields are Axiom A, Bull. Austral. Math. Soc. 51 (1995), 83-86. MR 95j:58092

[7] S. T. Liao, The qualitative theory of differential dynamical systems, Science Press, 1996. MR 98g:58041

[8] K. Moriyasu, The topological stability of diffeomorphisms, Nagoya Math. J. 123 (1991), 91102. MR 92g:58067

[9] J. Palis and W. de Melo, Geometric Theory of Dynamical systems, An Introduction, Springer, 1982. MR 84a:58004

[10] C. Pugh and C. Robinson, The $C^{1}$ closing lemma, including Hamiltonians, Ergod. Th. and Dynam. Sys. 3 (1983), 261-313. MR 85m:58106

[11] C. Robinson, Structural stability of $C^{1}$ flows, Dynamical systems-Warwick 1974 (ed. by A. Manning), Lecture Notes in Math. 468, Springer, 1975, 262-277. MR 58:31251

[12] C. Robinson, Stability theorems and hyperbolicity in dynamical systems, Rocky Mountain J. Math. 7 (1977), 425-437. MR 58:13200 
[13] K. Sakai, Diffeomorphisms with persistency, Proc. Amer. Math. Soc. 124 (1996), 2249-2254. MR 96i:58134

[14] K. Sakai, Topologically stable flows on surfaces, Far East J. Appl. Math. 1 (1997), 133-143.

[15] L. Wen, Combined two stabilities imply Axiom A for vector fields, Bull. Austral. Math. Soc. 48 (1993), 23-30. MR 94e:58073

[16] L. Wen, On the $C^{1}$ stability conjecture for flows, J. Diff. Eqs. 129 (1996), 334-357. MR 97j:58082

[17] L. Wen and Z. Xia, $C^{1}$ connecting lemmas, Trans. Amer. Math. Soc. 352 (2000), 5213-5230. MR 2001b:37024

Department of Mathematics, Tokushima University, Tokushima 770-8502, Japan

E-mail address: moriyasu@ias.tokushima-u.ac.jp

Department of Mathematics, Kanagawa University, Yokohama 221-8686, Japan

Current address: Department of Mathematics, Utsunomiya University, Mine-machi 321-8505, Japan

E-mail address: kazsaka@cc.kanagawa-u.ac.jp

E-mail address: sakaik01@kanagawa-u.ac.jp

Department of Mathematics, Tokyo Metropolitan University, Tokyo 192-0397, Japan

E-mail address: sumi@comp.metro-u.ac.jp 